\title{
Helicity-dependent generalized parton distributions and composite constituent quarks
}

\author{
Sergio Scopetta ${ }^{(a, b)}$ and Vicente Vento ${ }^{(b)}$ \\ (a) Dipartimento di Fisica, Università degli Studi di Perugia, via A. Pascoli 06100 Perugia, Italy, \\ and INFN, sezione di Perugia \\ (b) Departament de Fisica Tè̀rica, Universitat de València, 46100 Burjassot (València), Spain \\ and Institut de Física Corpuscular, Consejo Superior de Investigaciones Científicas
}

\begin{abstract}
An approach, recently proposed to calculate the nucleon generalized parton distributions (GPDs) in a constituent quark model (CQM) scenario, in which the constituent quarks are taken as complex systems, is used to obtain helicity-dependent GPDs. They are obtained from the wave functions of the non relativistic CQM of Isgur and Karl, convoluted with the helicity-dependent GPDs of the constituent quarks themselves. The latter are modelled by using the polarized structure functions of the constituent quark, the double distribution representation of GPDs, and a phenomenological constituent quark form factor. The present approach permits to access a kinematical range corresponding to both the Dokshitzer-Gribov-Lipatov-Altarelli-Parisi and the Efremov-Radyushkin-Brodsky-Lepage regions, for small values of the momentum transfer and of the skewedness parameter. In this kinematical region, the present calculation represents a prerequisite for the evaluation of cross sections relevant to deeply virtual Compton scattering. In particular, we have calculated the leading twist helicity-dependent GPD $\tilde{H}$ and, from our expressions,
\end{abstract}


its general relations with the non relativistic definition of the axial form factor and with the leading twist polarized quark density are consistently recovered.

Typeset using REVTEX 


\section{INTRODUCTION}

Generalized parton distributions (GPDs) [1-3] parametrize the non-perturbative hadron structure in hard exclusive processes (for a recent review, see, e.g., [4]). Their measurement would represent a unique way to access several crucial features of the structure of the nucleon, such as the Angular Momentum Sum Rule of the proton [3,5], as well as information on the structure of the proton in position space [6]. Therefore, relevant experimental efforts to measure GPDs, by means of exclusive electron Deep Inelastic Scattering (DIS) off the proton, are likely to take place in the next few years [7]. One of the most promising processes is represented by deeply virtual Compton scattering (DVCS) $[2,3,8]$.

At twist-two, four helicity-even GPDs occur. Two of them are usually called unpolarized GPDs and labeled $H$ and $E$. The first of them gives, in DIS kinematics, the usual quark density. The latter turns out to be a spin-flip distribution, in the sense that it implies a change of the spin of the target. The other two are usually called polarized or helicitydependent GPDs. They are labeled $\tilde{H}$ and $\tilde{E}$. The first of them yields in DIS kinematics the polarized quark density, the second is again a spin-flip distribution. The four of them enter the cross sections for the DVCS process, although the two non-spin-flip ones, $H$ and $\tilde{H}$, give the dominant contribution. Reasonable estimates are necessary for the planning of experiments.

For these quantities, several theoretical predictions have been already produced by using different descriptions of hadron structure: bag models [9], soliton models [10,11], lightfront [12,13] and Bethe Salpeter approaches [14], and phenomenological estimates based on parametrizations of parton distribution functions (PDFs) $[15,16]$. Besides, an impressive effort has been devoted to study the perturbative QCD evolution [17] of GPDs, and the GPDs at twist three accuracy [18]. Chiral-odd GPDs deserve a special mention: while they have been shown to be in principle experimentally accessible [19], no theoretical estimates are available for them.

We are interested in constituent quark model (CQM) calculations. In this framework, 
in order to compare model predictions with data taken in DIS experiments, one has to evolve, according to perturbative QCD, the leading twist component of the physical structure functions obtained at the low momentum scale associated with the model, from this so called "hadronic scale", $\mu_{0}^{2}[20,21]$, to the momentum scale of the data. Such a procedure has proven successful in describing the gross features of the standard PDFs by using different CQMs (see, e.g., [22]). Similar expectations motivated the study of GPDs in Ref. [23], where a simple formalism has been proposed to calculate the quark contribution to the GPDs from any non relativistic model. As an illustration, results for the GPD $H_{q}$, obtained in the non relativistic (NR) model of Isgur and Karl (IK) [24] have been evolved from $\mu_{0}^{2}$ up to DIS scales, to next to leading order (NLO) accuracy. In Ref. [25] the same quark contribution has been evaluated, at $\mu_{0}^{2}$, using the overlap representation of the GPDs [26] in light-front dynamics, along the lines developed in [27]. The same approach has been also applied to calculate the valence quark contribution to the helicity-dependent GPDs [28]. In all the papers dealing with CQM listed so far [23,25,28], only the Dokshitzer-GribovLipatov-Altarelli-Parisi (DGLAP) region of GPDs can be evaluated. To our knowledge, no CQM estimate of the helicity-dependent GPDs has been performed in the EfremovRadyushkin-Brodsky-Lepage (ERBL) region. This is precisely the argument of the present paper, where an approach recently proposed for the unpolarized GPDs in a CQM scenario [29], providing us with predictions also in the ERBL region, will be extended to the helicitydependent GPDs. In Ref. [29], the procedure of Ref. [23] has been extended and generalized since, as a matter of fact, the latter, when applied to the standard forward case, has been proven to reproduce only the gross features of PDFs [22]. In order to achieve a better agreement with data, the approach has to be improved. In a series of papers, it has been shown that unpolarized [30] and polarized [31] DIS data are consistent with a low energy scenario, dominated by complex constituent quarks inside the nucleon, defined through a scheme suggested by Altarelli, Cabibbo, Maiani and Petronzio (ACMP) [32], updated with modern phenomenological information. The same idea has been applied to demonstrate the evidence of complex objects inside the nucleon [33], analyzing intermediate energy data of 
electron scattering off the proton. A similar scenario, as old as the CQM itself [34], has been extensively used by other groups, starting form the concept of "valon" [35].

In Ref. [29], the description of the unpolarized forward case of Ref. [30] has been generalized and applied to the calculation scheme of Ref. [23]. As a result, more realistic predictions for the GPDs have been obtained and, at the same time, the ERBL region, not accessible before, has been explored. In particular, the evaluation of the sea quark contribution has become possible, so that GPDs can be calculated, in principle, in their full range of definition. Such an achievement will permit to estimate cross-sections that are relevant for actual GPDs measurements, providing us with an important tool for planning future experiments.

In here, the discussion is extended to helicity-dependent GPDs. The description of the polarized forward case of Ref. [31] is generalized to calculate helicity-dependent GPDs, following the path that lead to the results of Ref. [29] starting from the calculation of the unpolarized forward structure function in Ref. [30]. As in Ref. [29], the proposed approach will be applied here in a NR framework, which allows one to evaluate the GPDs only for small values of the 4-momentum transfer, $\Delta^{2}$ (corresponding to $\vec{\Delta}^{2} \ll m^{2}$, where $m$ is the constituent quark mass) and small values also for the skewedness parameter, $\xi$. The full kinematical range of definition of GPDs will be studied in a followup, introducing relativity in the scheme.

The paper is structured as follows. In the second section, after the definition of the main quantities of interest, a formula for the helicity-dependent current quark GPD $\tilde{H}_{q}$, in a CQM with point-like constituents, will be derived. For the same quantity, in the third section, in a scenario dominated by composite constituent quarks, a convolution formula in terms of the corresponding quantity of the constituent quark and of a polarized off-diagonal momentum distribution, will be derived in impulse approximation (IA). Then, the helicity-dependent constituent quark GPDs are built in the fourth section, according to ACMP philosophy and using the Double Distribution (DD's) representation [16,36,37] of the GPDs. In the fifth section, results obtained by using CQM wave functions of the IK model and our helicitydependent constituent quark GPDs will be shown. Conclusions will be drawn in the last 
section.

\section{CALCULATIONS OF HELICITY-DEPENDENT GPDS IN MODELS WITH POINT-LIKE QUARKS}

Let us consider a hard exclusive process, where the absorption of a high-energy virtual photon by a quark in a hadron target is followed by the emission of a particle to be later detected; finally, the interacting quark is reabsorbed back into the recoiling hadron. If the emitted and detected particle is, for example, a real photon, the so called Deeply Virtual Compton Scattering process takes place $[2,3,10]$. We adopt here the formalism used in Ref. [38]. Let us think of a nucleon target, with initial (final) momentum and helicity $P\left(P^{\prime}\right)$

and $s\left(s^{\prime}\right)$, respectively. The GPDs $\tilde{H}_{q}\left(x, \xi, \Delta^{2}\right)$ and $\tilde{E}_{q}\left(x, \xi, \Delta^{2}\right)$ are defined through the expression

$$
\begin{aligned}
\tilde{F}_{s^{\prime} s}^{q}\left(x, \xi, \Delta^{2}\right) & =\frac{1}{2} \int \frac{d \lambda}{2 \pi} e^{i \lambda x}\left\langle P^{\prime} s^{\prime}\left|\bar{\psi}_{q}\left(-\frac{\lambda n}{2}\right) \not h \gamma_{5} \psi_{q}\left(\frac{\lambda n}{2}\right)\right| P s\right\rangle= \\
& =\tilde{H}_{q}\left(x, \xi, \Delta^{2}\right) \frac{1}{2} \bar{U}\left(P^{\prime}, s^{\prime}\right) h \gamma_{5} U(P, s)+\tilde{E}_{q}\left(x, \xi, \Delta^{2}\right) \frac{1}{2} \bar{U}\left(P^{\prime}, s^{\prime}\right) \frac{\Delta \cdot n \gamma_{5}}{2 M} U(P, s)
\end{aligned}
$$

where $\Delta=P^{\prime}-P$ is the 4 -momentum transfer to the nucleon, $\psi_{q}$ is the quark field and $\mathrm{M}$ is the nucleon mass. It is convenient to work in a system of coordinates where the photon 4-momentum, $q^{\mu}=\left(q_{0}, \vec{q}\right)$, and $\bar{P}=\left(P+P^{\prime}\right) / 2$ are collinear along $z$. The $\xi$ variable in the arguments of the GPDs is the so called "skewedness", parametrizing the asymmetry of the process. It is defined by the relation $\xi=-n \cdot \Delta / 2$, where $n$ is a light-like 4 -vector satisfying the condition $n \cdot \bar{P}=1$. As explained in [3], GPDs describe the amplitude for finding a quark with momentum fraction $x+\xi$ (in the Infinite Momentum Frame) in a nucleon with momentum $(1+\xi) \bar{P}$ and replacing it back into the nucleon with a momentum transfer $\Delta$. Besides, when the quark longitudinal momentum fraction $x$ of the average nucleon momentum $\bar{P}$ is less than $-\xi$, GPDs describe antiquarks; when it is larger than $\xi$, they describe quarks; when it is between $-\xi$ and $\xi$, they describe $q \bar{q}$ pairs. The first and 
second $x$ intervals are commonly referred to as the DGLAP region and the third as the ERBL region [38], following the pattern of evolution in the factorization scale. One should keep in mind that, besides the variables $x, \xi$ and $\Delta^{2}$ explicitly shown, GPDs depend, as the standard PDFs, on the momentum scale $Q^{2}$ at which they are measured or calculated. To make the presentation easier looking, we omit the latter dependence unless when specifically needed. The values of $\xi$ which are possible for a given value of $\Delta^{2}$ are:

$$
0 \leq \xi \leq \sqrt{-\Delta^{2}} / \sqrt{4 M^{2}-\Delta^{2}}
$$

The known constraints of $\tilde{H}_{q}\left(x, \xi, \Delta^{2}\right)$ are:

i) the so called "forward" limit, $P^{\prime}=P$, i.e., $\Delta^{2}=\xi=0$, yields the usual polarized PDFs

$$
\tilde{H}_{q}(x, 0,0)=\Delta q(x)
$$

ii) the integration over $x$, yields the contribution of the quark of flavor $q$ to the axial form factor (ff) of the target $A$, called hereafter $G_{A}^{q}\left(\Delta^{2}\right)$ :

$$
\int d x \tilde{H}_{q}\left(x, \xi, \Delta^{2}\right)=G_{A}^{q}\left(\Delta^{2}\right)
$$

iii) the polynomiality property [38], according to which the $x$-integrals of $x^{n} \tilde{H}^{q}$ and of $x^{n} \tilde{E}^{q}$ are polynomials in $\xi$ of order $n+1$.

In $[23,29]$, the IA expression for the unpolarized $H_{q}\left(x, \xi, \Delta^{2}\right)$, suitable to perform CQM calculations, was obtained. In a similar fashion, we derive next a formula for the helicitydependent GPD $\tilde{H}_{q}\left(x, \xi, \Delta^{2}\right)$ from the definition (1).

Using the light-cone spinor definitions as given in the appendix B of [4], and defining: $k^{+}=\left(k_{0}+k_{3}\right) / \sqrt{2}, \vec{k}_{\perp}=\left(k_{1}, k_{2}\right)$, for the light-cone helicity combination $s^{\prime} s=\frac{1}{2} \frac{1}{2}=++$ one obtains

$$
\tilde{F}_{++}^{q}\left(x, \xi, \Delta^{2}\right)=\sqrt{1-\xi^{2}} \tilde{H}_{q}\left(x, \xi, \Delta^{2}\right)-\frac{\xi^{2}}{\sqrt{1-\xi^{2}}} \tilde{E}_{q}\left(x, \xi, \Delta^{2}\right)
$$

so that, for $\xi^{2} \ll 1$ : 


$$
\tilde{F}_{++}^{q}\left(x, \xi, \Delta^{2}\right)=\tilde{H}_{q}\left(x, \xi, \Delta^{2}\right)-\xi^{2}\left(\frac{1}{2} \tilde{H}_{q}\left(x, \xi, \Delta^{2}\right)+\tilde{E}_{q}\left(x, \xi, \Delta^{2}\right)\right)+O\left(\xi^{4}\right),
$$

i.e.

$$
\tilde{F}_{++}^{q}\left(x, \xi, \Delta^{2}\right)=\tilde{H}_{q}\left(x, \xi, \Delta^{2}\right)+O\left(\xi^{2}\right) .
$$

According to this equation, in order to obtain the $\operatorname{GPD} \tilde{H}_{q}\left(x, \xi, \Delta^{2}\right)$ for $\xi^{2} \ll 1$ one has to evaluate $\tilde{F}_{++}^{q}$, starting from its definition, Eq (1). Using light-cone quantized quark fields in the l.h.s., whose creation and annihilation operators, $b^{\dagger}(k)$ and $b(k)$, obey the commutation relation

$$
\left\{b\left(k^{\prime}\right), b^{\dagger}(k)\right\}=(2 \pi)^{3} 2 k^{+} \delta\left(k^{\prime+}-k^{+}\right) \delta^{2}\left(\vec{k}_{\perp}-\vec{k}_{\perp}^{\prime}\right),
$$

and using light-cone states normalized as

$$
\left\langle P^{\prime} \mid P\right\rangle=(2 \pi)^{3} 2 P^{+} \delta\left(P^{\prime+}-P^{+}\right) \delta^{2}\left(\vec{P}_{\perp}-\vec{P}_{\perp}^{\prime}\right)
$$

one obtains, for $x>\xi[38]$

$$
\tilde{F}_{++}^{q}\left(x, \xi, \Delta^{2}\right)=\frac{1}{2 \bar{P}^{+} V} \int \frac{d^{2} k_{\perp}}{2 \sqrt{\left|x^{2}-\xi^{2}\right|}(2 \pi)^{3}}\left\langle b^{\dagger}(k+\Delta) b(k)\right\rangle,
$$

where

$$
\left\langle b^{\dagger}\left(k^{\prime}\right) b(k)\right\rangle=\sum_{\lambda} \operatorname{sign}(\lambda)\left\langle P^{\prime}+\left|b_{\lambda}^{\dagger}\left((x-\xi) \bar{P}^{+}, \vec{k}_{\perp}^{\prime}\right) b_{\lambda}\left((x+\xi) \bar{P}^{+}, \vec{k}_{\perp}\right)\right| P+\right\rangle,
$$

and $V$ is a volume factor. Since we want to obtain only the quark contribution to $\tilde{H}_{q}$, the only one which can be evaluated in a CQM with three valence, point-like quarks, we are interested here in the $x>\xi$ region.

Eq. (10) can be written (see [29] where all the steps of a similar derivation are explicitely given):

$$
\tilde{F}_{++}^{q}\left(x, \xi, \Delta^{2}\right)=\frac{1}{2 \bar{P}^{+} V} \int \frac{d^{2} k_{\perp} d k^{+}}{2 \sqrt{k^{+} k^{\prime+}}(2 \pi)^{3}} \delta\left(\frac{k^{+}}{\bar{P}^{+}}-(x+\xi)\right)\left\langle b^{\dagger}(k+\Delta) b(k)\right\rangle .
$$

In a NR framework, states and creation and annihilation operators have to be normalized according to 


$$
\left\langle\vec{P}^{\prime} \mid \vec{P}\right\rangle=(2 \pi)^{3} \delta\left(P^{\prime+}-P^{+}\right) \delta\left(\vec{P}_{\perp}^{\prime}-\vec{P}_{\perp}\right)
$$

and

$$
\left\{b\left(k^{\prime+}, \vec{k}_{\perp}\right), b^{\dagger}\left(k^{+}, \vec{k}_{\perp}^{\prime}\right)\right\}=(2 \pi)^{3} \delta\left(k^{\prime+}-k^{+}\right) \delta\left(\vec{k}_{\perp}^{\prime}-\vec{k}_{\perp}\right)
$$

respectively. As a consequence, one has to consider that [39]:

$$
\begin{gathered}
|P\rangle \rightarrow \sqrt{2 P^{+}}|\vec{P}\rangle, \\
b(k) \rightarrow \sqrt{2 k^{+}} b\left(k^{+}, \vec{k}_{\perp}\right),
\end{gathered}
$$

so that in Eq. (12), in terms of the new states and fields, one has to perform the substitution

$$
\begin{aligned}
\left\langle b^{\dagger}(k+\Delta) b(k)\right\rangle= & \sum_{\lambda} \operatorname{sign}(\lambda)\left\langle P^{\prime}+\left|b_{\lambda}^{\dagger}\left((x-\xi) \bar{P}^{+}, \vec{k}_{\perp}+\vec{\Delta}_{\perp}\right) b_{\lambda}\left((x+\xi) \bar{P}^{+}, \vec{k}_{\perp}\right)\right| P+\right\rangle \rightarrow \\
\rightarrow & 2 \sqrt{1-\xi^{2}} \bar{P}^{+} \sqrt{2 k^{\prime+} 2 k^{+}} \times \\
& \times \sum_{\lambda} \operatorname{sign}(\lambda)\left\langle\vec{P}^{\prime}\left|b_{\lambda}^{\dagger}\left(k^{+}+\Delta^{+}, \vec{k}_{\perp}+\vec{\Delta}_{\perp}\right) b_{\lambda}\left(k^{+}, \vec{k}_{\perp}\right)\right| \vec{P}\right\rangle
\end{aligned}
$$

where the relation $2 \sqrt{1-\xi^{2}} \bar{P}^{+}=\sqrt{2 P^{+}} \sqrt{2 P^{\prime+}}$ has been used.

Now, inserting Eq. (17) in Eq. (12), one gets

$$
\begin{aligned}
\tilde{F}_{++}^{q}\left(x, \xi, \Delta^{2}\right) & =\frac{1}{V} \int \frac{d^{2} k_{\perp} d k^{+}}{2 \sqrt{k^{+} k^{\prime+}}(2 \pi)^{3}} \delta\left(\frac{k^{+}}{\bar{P}^{+}}-(x+\xi)\right) \sqrt{1-\xi^{2}} 2 \sqrt{k^{+} k^{++}} \times \\
& \times \sum_{\lambda} \operatorname{sign}(\lambda)\left\langle\vec{P}^{\prime}\left|b_{\lambda}^{\dagger}\left(k^{+}+\Delta^{+}, \vec{k}_{\perp}+\vec{\Delta}_{\perp}\right) b_{\lambda}\left(k^{+}, \vec{k}_{\perp}\right)\right| \vec{P}\right\rangle= \\
& =\frac{1}{V} \int \frac{d^{2} k_{\perp} d k^{+}}{(2 \pi)^{3}} \delta\left(\frac{k^{+}}{\bar{P}^{+}}-(x+\xi)\right) \times \\
& \times \sum_{\lambda} \operatorname{sign}(\lambda)\left\langle\vec{P}^{\prime}\left|b_{\lambda}^{\dagger}\left(k^{+}+\Delta^{+}, \vec{k}_{\perp}+\vec{\Delta}_{\perp}\right) b_{\lambda}\left(k^{+}, \vec{k}_{\perp}\right)\right| \vec{P}\right\rangle+O\left(\xi^{2}\right) .
\end{aligned}
$$

The constituent quarks with mass $m$ are taken to be on shell, $k_{0}=\sqrt{\vec{k}^{2}+m^{2}}$, thus

$$
d^{2} k_{\perp} d k^{+} \rightarrow\left(\frac{k^{+}}{k_{0}}\right) d \vec{k}
$$

and from Eq. (18) and Eq. (7), one gets 


$$
\begin{aligned}
\tilde{H}_{q}\left(x, \xi, \Delta^{2}\right) & =\int d \vec{k} \delta\left(\frac{k^{+}}{\bar{P}^{+}}-(x+\xi)\right) \times \\
& \times\left[\frac{1}{(2 \pi)^{3} V} \frac{k^{+}}{k_{0}} \sum_{\lambda} \operatorname{sign}(\lambda)\left\langle\vec{P}^{\prime}\left|b_{\lambda}^{\dagger}\left(k^{+}+\Delta^{+}, \vec{k}_{\perp}+\vec{\Delta}_{\perp}\right) b_{\lambda}\left(k^{+}, \vec{k}_{\perp}\right)\right| \vec{P}\right\rangle\right]+O\left(\xi^{2}\right)= \\
& =\int d \vec{k} \delta\left(\frac{k^{+}}{\bar{P}^{+}}-(x+\xi)\right)\left[\tilde{n}_{q}(\vec{k}, \vec{k}+\vec{\Delta})+O\left(\frac{\vec{k}^{2}}{m^{2}} \frac{(\vec{k}+\vec{\Delta})^{2}}{m^{2}}\right)\right]+O\left(\xi^{2}\right) .
\end{aligned}
$$

In the last step, the definition of a (non-diagonal) polarized NR momentum distribution, $\tilde{n}_{q}(\vec{k}, \vec{k}+\vec{\Delta})$, has been used, together with the fact that a NR momentum distribution describes the probability of finding a constituent of momentum $\vec{k}$ in a given system up to terms of order $\frac{\vec{k}^{2}}{m^{2}}[40]$.

So far, we have shown that, in a NR CQM, the GPD $\tilde{H}_{q}\left(x, \xi, \Delta^{2}\right)$ can be calculated, for $\xi^{2} \ll 1, \vec{k}^{2} \ll m^{2}$ and $(\vec{k}+\vec{\Delta})^{2} \ll m^{2}$ (which means, in turn, $\vec{\Delta}^{2} \ll m^{2}$ ), through the following expression:

$$
\tilde{H}_{q}\left(x, \xi, \Delta^{2}\right)=\int d \vec{k} \delta\left(\frac{k^{+}}{\bar{P}^{+}}-(x+\xi)\right) \tilde{n}_{q}(\vec{k}, \vec{k}+\vec{\Delta})+O\left(\xi^{2}, \frac{\vec{k}^{2}}{m^{2}}, \frac{\vec{\Delta}^{2}}{m^{2}}\right) .
$$

The above equation, corresponding to Eq. (8) in [23] if polarization is taken into account, permits the calculation of $\tilde{H}_{q}\left(x, \xi, \Delta^{2}\right)$ in any CQM, and it naturally verifies some of the properties of the GPDs. In fact, the polarized quark density, $\Delta q(x)$, as obtained by analyzing, in IA, the DIS off the nucleon (see, e.g., [41]), assuming that the interacting quark is on-shell, is recovered in the forward limit, where $\Delta^{2}=0, \xi=0$ :

$$
\Delta q(x)=\tilde{H}_{q}(x, 0,0)=\int d \vec{k} \tilde{n}_{q}(\vec{k}) \delta\left(x-\frac{k^{+}}{P^{+}}\right),
$$

so that the constraint Eq. (3) is fulfilled. In the above equation, $\tilde{n}_{q}(\vec{k})$ is the NR polarized momentum distribution of the quarks in the nucleon and, integrating Eq. (22) over $x$, one obtains the contribution of the quark of flavor $q$ to the IA definition of the axial ff, so that Eq. (4) is verified.

The polynomiality condition is formally fulfilled by the GPD defined in Eq. (22), although the present accuracy of the model, explicitly written in the latter equation, does not allow to really check polynomiality, due to the effects of the Munich Symmetry [42], which excludes $O(\xi)$ contributions. 
With respect to Eq. (22), a few caveats, already listed in Ref. [29] for the unpolarized case, are necessary.

i) One should keep in mind that Eq. (22) is a NR result, holding for $\xi^{2} \ll 1$, under the conditions $\vec{k}^{2} \ll m^{2}, \vec{\Delta}^{2} \ll m^{2}$. If one wants to treat more general processes, the NR limit should be relaxed by taking into account relativistic corrections. In this way, at the same time, an expression to evaluate $\tilde{E}_{q}\left(x, \xi, \Delta^{2}\right)$ could be obtained.

ii) The Constituent Quarks are point-like objects.

iii) If use is made of a CQM, containing only constituent quarks (and also antiquarks in the case of mesons), only the quark (and antiquark) contribution to the GPDs can be evaluated, i.e., only the region $x \geq \xi$ (and also $x \leq-\xi$ for mesons) can be explored. In order to introduce the study of the ERBL region $(-\xi \leq x \leq \xi)$, so that observables like cross-sections, spin asymmetries and so on can be calculated, the model has to be improved.

iv) In actual calculations, the evaluation of Eq. (22) requires the choice of a reference frame. In the following, the Breit Frame will be chosen, where one has $\Delta^{2}=-\vec{\Delta}^{2}$ and, in the NR limit we are studying, one finds $\sqrt{2} \bar{P}^{+} \rightarrow M$. It happens therefore that, in the argument of the $\delta$ function in Eq. (22), the $x$ variable for the valence quarks is not defined in its natural support, i.e. it can be larger than 1 and smaller than $\xi$. Although the support violation is small for the calculations that will be shown here, it has to be reported as a drawback of the approach.

The issue iii) will be discussed in the next sections, by relaxing the condition ii) and allowing for a finite size and composite structure of the constituent quark.

\section{HELICITY- DEPENDENT GPDS IN A CONSTITUENT QUARK SCENARIO}

In the standard forward case, the procedure described in the previous section, has been proven to be able to reproduce the gross features of PDFs [22]. In order to achieve a better agreement with data, the approach has to be enriched.

In previous papers, it has been shown that unpolarized [30] and polarized [31] DIS 
data are consistent with a low energy scenario dominated by complex systems of point-like partons. The forward parton distributions are therefore given by a convolution between constituent quark momentum distributions and constituent quark structure functions. The latter quantities were obtained by using updated phenomenological information in a scenario firstly suggested by Altarelli, Cabibbo, Maiani and Petronzio (ACMP) already in the seventies [32]. Following the same idea, in Ref. [29] a model for the reaction mechanism of an off-forward process, such as DVCS, where GPDs could be measured, has been proposed. As a result, a convolution formula giving the proton $H_{q}$ GPD in terms of a constituent quark offforward momentum distribution, $H_{q_{0}}$, and of a GPD of the constituent quark $q_{0}$ itself, $H_{q_{0} q}$, has been derived. In here, a similar program will be developed for the helicity-dependent $\operatorname{GPD} \tilde{H}_{q}$.

It is assumed that the hard scattering with the virtual photon takes place on a parton of a spin $1 / 2$ target, such as the proton, made of complex constituents. The scenario is depicted in Fig. 1 for the special case of DVCS, in the handbag approximation. One parton (current quark) with momentum $k$, belonging to a given constituent of momentum p, interacts with the probe and it is afterwards reabsorbed, with momentum $k+\Delta$, by the same constituent, without further re-scattering with the recoiling system of momentum $P_{R}$. We suggest here an analysis of the process which is quite similar to the usual IA approach to DIS off nuclei [40], recently applied also to nuclear GPDs [43].

In the class of frames chosen in section 2, and in addition to the kinematical variables, $x$ and $\xi$ one needs a few more to describe the process. In particular, $x^{\prime}$ and $\xi^{\prime}$, for the "internal" target, i.e., the constituent quark, have to be introduced. These quantities can be obtained defining the "+" components of the momentum $k$ and $k+\Delta$ of the struck parton before and after the interaction, with respect to $\bar{P}^{+}$and $\bar{p}^{+}=\frac{1}{2}\left(p+p^{\prime}\right)^{+}$:

$$
\begin{aligned}
k^{+} & =(x+\xi) \bar{P}^{+}=\left(x^{\prime}+\xi^{\prime}\right) \bar{p}^{+}, \\
(k+\Delta)^{+} & =(x-\xi) \bar{P}^{+}=\left(x^{\prime}-\xi^{\prime}\right) \bar{p}^{+} .
\end{aligned}
$$

From the above expressions, $\xi^{\prime}$ and $x^{\prime}$ are immediately obtained as 


$$
\begin{aligned}
& \xi^{\prime}=-\frac{\Delta^{+}}{2 \bar{p}^{+}} \\
& x^{\prime}=\frac{\xi^{\prime}}{\xi} x
\end{aligned}
$$

and, since $\xi=-\Delta^{+} /\left(2 \bar{P}^{+}\right)$, if $\tilde{z}=p^{+} / P^{+}$, one also has

$$
\xi^{\prime}=\frac{\xi}{\tilde{z}(1+\xi)-\xi} .
$$

In order to derive a convolution formula in IA for $\tilde{H}_{q}$, the procedure used for standard DIS will be adopted [40]. First, in Eq. (20), two complete sets of states, corresponding to the interacting particle and to the recoiling system, are properly inserted to the left and right-hand sides of the quark operator:

$$
\begin{aligned}
\tilde{H}_{q}\left(x, \xi, \Delta^{2}\right)= & \left\langleP ^ { \prime } S \left|\sum_{P_{R}^{\prime}, S_{R}^{\prime}, p^{\prime}, s^{\prime}}\left\{\left|P_{R}^{\prime} S_{R}^{\prime}\right\rangle\left|p^{\prime} s^{\prime}\right\rangle\right\}\left\{\left\langle P_{R}^{\prime} S_{R}^{\prime}\right|\left\langle p^{\prime} s^{\prime}\right|\right\}\right.\right. \\
& \int \frac{d \vec{k}}{(2 \pi)^{3}} \frac{k^{+}}{k_{0}} \delta\left(\frac{k^{+}}{\bar{P}^{+}}-(x+\xi)\right) \frac{1}{V} \sum_{\lambda} \operatorname{sign}(\lambda) b_{q, \lambda}^{\dagger}\left(k^{+}+\Delta^{+}, \vec{k}_{\perp}+\vec{\Delta}_{\perp}\right) b_{q, \lambda}\left(k^{+}, \vec{k}_{\perp}\right) \\
& \sum_{P_{R}, S_{R}, p, s}\left\{\left|P_{R} S_{R}\right\rangle|p s\rangle\right\}\left\{\left\langle P_{R} S_{R}|\langle p s|\} \mid P S\right\rangle ;\right.
\end{aligned}
$$

second, since eventually use has to be made of NR wave functions, a state $|\vec{p}\rangle$ has to be normalized in a NR manner:

$$
\left\langle\vec{p}^{\prime} \mid \vec{p}\right\rangle=(2 \pi)^{3} \delta\left(\vec{p}^{\prime}-\vec{p}\right)
$$

so that in Eq. (29) one has to perform the substitution:

$$
\left|p>\rightarrow \sqrt{\frac{p^{+}}{p^{0}}}\right| \vec{p}>.
$$

Since, using IA in the intrinsic frame of the target one has, for the NR states:

$$
\left\{\left\langle\vec{P}_{R} S_{R}|\langle\vec{p} s|\} \mid \vec{P} S\right\rangle=\left\langle\vec{P}_{R} S_{R}, \vec{p} s \mid \vec{P} S\right\rangle(2 \pi)^{3} \delta^{3}\left(\vec{P}-\vec{P}_{R}-\vec{p}\right) \delta_{S, S_{R} s}\right.
$$

a convolution formula is readily obtained from Eq. (29):

$$
\begin{aligned}
\tilde{H}_{q}\left(x, \xi, \Delta^{2}\right) & \simeq \sum_{q_{0}} \int d E \int d \vec{p} \sqrt{\frac{p^{+}(p+\Delta)^{+}}{p_{0}(p+\Delta)_{0}}} \tilde{P}_{q_{0}}(\vec{p}, \vec{p}+\vec{\Delta}, E) \frac{\xi^{\prime}}{\xi} \tilde{H}_{q_{0} q}\left(x^{\prime}, \xi^{\prime}, \Delta^{2}\right)+\mathcal{O}\left(\xi^{2}\right)= \\
& =\sum_{q_{0}} \int d E \int d \vec{p}\left[\tilde{P}_{q_{0}}(\vec{p}, \vec{p}+\vec{\Delta}, E)+\mathcal{O}\left(\vec{p}^{2} / M^{2}, \vec{\Delta}^{2} / M^{2}\right)\right] \\
& \times \frac{\xi^{\prime}}{\xi} \tilde{H}_{q_{0} q}\left(x^{\prime}, \xi^{\prime}, \Delta^{2}\right)+\mathcal{O}\left(\xi^{2}\right) .
\end{aligned}
$$


In the above equations, the kinetic energies of the residual system and of the recoiling target have been neglected, and $\tilde{P}_{q_{0}}(\vec{p}, \vec{p}+\vec{\Delta}, E)$ is a spin dependent, off-diagonal spectral function for the constituent quark in the target:

$$
\begin{aligned}
\tilde{P}_{q_{0}}(\vec{p}, \vec{p}+\vec{\Delta}, E) & =\frac{1}{(2 \pi)^{3}} \sum_{R, s} \operatorname{sign}(s)\left\langle\vec{P}^{\prime} M \mid(\vec{P}-\vec{p}) S_{R},(\vec{p}+\vec{\Delta}) s\right\rangle\left\langle(\vec{P}-\vec{p}) S_{R}, \vec{p} s \mid \vec{P} M\right\rangle \times \\
& \times \delta\left(E-E_{R}^{*}\right) .
\end{aligned}
$$

Besides, the quantity

$$
\begin{aligned}
\tilde{H}_{q_{0} q}\left(x^{\prime}, \xi^{\prime}, \Delta^{2}\right) & =\int d \vec{k} \delta\left(\frac{k^{+}}{\bar{p}^{+}}-\left(x^{\prime}+\xi^{\prime}\right)\right) \\
& \times \frac{1}{(2 \pi)^{3} V} \frac{k^{+}}{k_{0}} \sum_{\lambda} \operatorname{sign}(\lambda)\left\langle p^{\prime}\left|b_{q, \lambda}^{\dagger}\left(k^{+}+\Delta^{+}, \vec{k}_{\perp}+\vec{\Delta}_{\perp}\right) b_{q, \lambda}\left(k^{+}, \vec{k}_{\perp}\right)\right| p\right\rangle= \\
& =\frac{\xi}{\xi^{\prime}} \int d \vec{k} \delta\left(\frac{k^{+}}{\bar{P}^{+}}-(x+\xi)\right) \\
& \times \frac{1}{(2 \pi)^{3} V} \frac{k^{+}}{k_{0}} \sum_{\lambda} \operatorname{sign}(\lambda)\left\langle p^{\prime}\left|b_{q, \lambda}^{\dagger}\left(k^{+}+\Delta^{+}, \vec{k}_{\perp}+\vec{\Delta}_{\perp}\right) b_{q, \lambda}\left(k^{+}, \vec{k}_{\perp}\right)\right| p\right\rangle
\end{aligned}
$$

is, according to Eq. (20), the helicity-dependent GPD of the constituent quark $q_{0}$ up to terms of order $O\left(\xi^{2}\right)$, and in the above equation use has been made of Eqs. (27) and (28).

The delta function in $\mathrm{Eq}(34)$ defines $E$, the removal energy, in terms of $E_{R}^{*}$, the excitation energy of the recoiling system.

Concerning Eqs (33-35), two comments are in order.

The first concerns the accuracy of the actual calculations which will be presented. In the following, NR wave functions will be used to evaluate Eq. (33), so that the accuracy of the calculation is of order $\mathcal{O}\left(\vec{p}^{2} / M^{2}, \vec{\Delta}^{2} / M^{2}\right)$. In fact, if use is made of $\mathrm{NR}$ wave functions to calculate the non-diagonal spectral function, the result holds for $\vec{p}^{2},(\vec{p}+\vec{\Delta})^{2}<<M^{2}$. The same constraint can be written $\vec{p}^{2}, \vec{\Delta}^{2}<<M^{2}$.

Second, from the study of forward DIS off nuclei, it is known that, in going from a covariant formalism to one where use can be made of the usual NR wave functions, one has to keep the correct normalization [40]. This procedure leads to the appearance of the "flux factor", represented in Eq. (32) by the expression $\sqrt{p^{+}(p+\Delta)^{+} / p_{0}(p+\Delta)_{0}}$ (which gives, in forward DIS, the usual $p^{+} / p_{0}$ term). This factor gives one at order $\mathcal{O}\left(\vec{p}^{2} / M^{2}, \vec{\Delta}^{2} / M^{2}\right)$, 
which is the accuracy of the present analysis, and it will not be included in the calculation described below. If one wants to be predictive in more general processes at higher momentum transfer, the accuracy of Eq. (33) is not good enough any more and the calculation, in order to be consistent, has to be performed taking into account relativistic corrections.

Eq. (33) can be written in the form

$$
\begin{aligned}
\tilde{H}_{q}\left(x, \xi, \Delta^{2}\right) & =\sum_{q_{0}} \int d E \int d \vec{p} \tilde{P}_{q_{0}}(\vec{p}, \vec{p}+\vec{\Delta}) \frac{\xi^{\prime}}{\xi} \tilde{H}_{q_{0} q}\left(x^{\prime}, \xi^{\prime}, \Delta^{2}\right)= \\
& =\sum_{q_{0}} \int_{x}^{1} \frac{d z}{z} \int d E \int d \vec{p} \tilde{P}_{q_{0}}(\vec{p}, \vec{p}+\vec{\Delta}) \delta\left(z-\frac{\xi}{\xi^{\prime}}\right) \tilde{H}_{q_{0} q}\left(\frac{x}{z}, \frac{\xi}{z}, \Delta^{2}\right) .
\end{aligned}
$$

Taking into account that

$$
z-\frac{\xi}{\xi^{\prime}}=z-[\tilde{z}(1+\xi)-\xi]=z+\xi-\frac{p^{+}}{P^{+}}(1+\xi)=z+\xi-\frac{p^{+}}{\bar{P}^{+}}
$$

Eq. (36) can also be written in the form:

$$
\tilde{H}_{q}\left(x, \xi, \Delta^{2}\right)=\sum_{q_{0}} \int_{x}^{1} \frac{d z}{z} \tilde{H}_{q_{0}}\left(z, \xi, \Delta^{2}\right) \tilde{H}_{q_{0} q}\left(\frac{x}{z}, \frac{\xi}{z}, \Delta^{2}\right)
$$

where

$$
\tilde{H}_{q_{0}}\left(z, \xi, \Delta^{2}\right)=\int d E \int d \vec{p} \tilde{P}_{q_{0}}(\vec{p}, \vec{p}+\vec{\Delta}) \delta\left(z+\xi-\frac{p^{+}}{\bar{P}^{+}}\right)
$$

One should notice that Eqs. (38) and (39) or, which is the same, Eq. (33), fulfill the constraint $i)-i i i)$ previously listed.

The constraint $i$ ), i.e. the forward limit of GPDs, is verified. In fact, by taking the forward limit $\left(\Delta^{2} \rightarrow 0, \xi \rightarrow 0\right)$ of Eq. (38), one gets the expression which is usually found, for the polarized parton distribution $\Delta q(x)$, in the IA analysis of polarized DIS off polarized three-body systems made of composite constituents (see, i.e., [44] for the ${ }^{3}$ He target)

$$
\Delta q(x)=\tilde{H}_{q}(x, 0,0)=\sum_{q_{0}} \int_{x}^{1} \frac{d z}{z} g_{q_{0}}(z) \Delta q_{q_{0}}\left(\frac{x}{z}\right)
$$

In the latter equation,

$$
g_{q_{0}}(z)=\tilde{H}_{q_{0}}(z, 0,0)=\int d E \int d \vec{p} \tilde{P}_{q_{0}}(\vec{p}, E) \delta\left(z-\frac{p^{+}}{P^{+}}\right)
$$


is the light-cone momentum distribution of the constituent $q_{0}$ in the nucleon, $\Delta q_{q_{0}}(x)=$ $\tilde{H}_{q_{0} q}(x, 0,0)$ is the distribution of the quark of flavour $q$ in the constituent $q_{0}$ and $\tilde{P}_{q_{0}}(\vec{p}, E)$, the $\Delta^{2} \longrightarrow 0$ limit of Eq. (36), is the one body spin dependent spectral function, labeled $P_{\|}$ in Ref. $[45,44]$. If the system were a three-body nucleus, the constituents would be nucleons and Eqs. (38)-(41) would coincide with the corresponding ones obtained in Ref. [44] for the polarized ${ }^{3} \mathrm{He}$ target.

The constraint $i i)$, i.e. the $x$-integral of the GPD $\tilde{H}_{q}$, is also naturally fulfilled. In fact, by $x$-integrating Eq. (38), one easily obtains:

$$
\begin{aligned}
\int d x \tilde{H}_{q}\left(x, \xi, \Delta^{2}\right) & =\sum_{q_{0}} \int d x \int \frac{d z}{z} \tilde{H}_{q_{0}}\left(z, \xi, \Delta^{2}\right) \tilde{H}_{q_{0} q}\left(\frac{x}{z}, \frac{\xi}{z}, \Delta^{2}\right)= \\
& =\sum_{q_{0}} \int d x^{\prime} \tilde{H}_{q_{0} q}\left(x^{\prime}, \xi^{\prime}, \Delta^{2}\right) \int d z \tilde{H}_{q_{0}}\left(z, \xi, \Delta^{2}\right)= \\
& =\sum_{q_{0}} G_{q_{0}}^{q}\left(\Delta^{2}\right) G_{p}^{q_{0}}\left(\Delta^{2}\right)=G_{p}^{q}\left(\Delta^{2}\right) .
\end{aligned}
$$

In the equation above, $G_{p}^{q}\left(\Delta^{2}\right)$ is the contribution, of the quark of flavour $q$, to the proton axial ff; $G_{q_{0}}^{q}\left(\Delta^{2}\right)$ is the contribution, of the quark of flavour $q$, to the constituent quark $q_{0}$ axial ff; $G_{q_{0}}^{p}\left(\Delta^{2}\right)$ is a proton axial "pointlike ff", which would represent the contribution of the quark $q_{0}$ to the axial $\mathrm{ff}$ of the proton if $q_{0}$ were point-like. $G_{p}^{q_{0}}\left(\Delta^{2}\right)$ is given, in the present approximation, by

$$
G_{p}^{q_{0}}\left(\Delta^{2}\right)=\int d E \int d \vec{p} \tilde{P}_{q_{0}}(\vec{p}, \vec{p}+\vec{\Delta}, E)=\int d z \tilde{H}_{q_{0}}\left(z, \xi, \Delta^{2}\right) .
$$

Eventually the polynomiality, condition iii), is formally fulfilled by Eq. (33), although one should always remember that it is a result of order $\mathcal{O}\left(\xi^{2}\right)$, so that high moments cannot be really checked.

Summarizing, an IA convolution formula has been derived, valid for any helicitydependent GPD $\tilde{H}_{q}$ of a spin $1 / 2$ hadron target made of three spin $1 / 2$ constituents, Eq. (33) (or, which is equivalent, Eq. (38)), at order $\mathcal{O}\left(\frac{\vec{p}^{2}}{M^{2}}, \frac{\vec{\Delta}^{2}}{M^{2}}, \xi^{2}\right)$, in terms of a spin-dependent, non-diagonal spectral function, Eq. (34), and of the helicity-dependent GPD $\tilde{H}_{q_{0} q}$ of the internal target. Convolution formulae are usually obtained in describing quantities, such as structure functions, which have a probabilistic interpretation. This is the case of Eq. 
(40). However, GPDs represent amplitudes and not probability densities. Our convolution equation for GPDs, obtained as the result of a mathematical procedure, also used by several authors in the context of the IA in nuclear GPDs $[46,47,43]$, has a different meaning. It involves wave functions evaluated for different values of the momentum of one of the particles (one body off diagonal matrix elements). Besides, in the derivation, the simple structure of the final result, Eq. (38), is due to the restrictions imposed in the kinematics $\left(\Delta^{2} \ll m^{2}, \xi^{2} \ll 1\right)$. Had these approximations, which are beyond IA, not been made, Eq. (38) would be more complicated. For example, a contamination from the GPD $E_{q}$ would be found. Therefore, the formulae we are discussing here are not strict convolution formulae in the conventional probabilistic sense. Finally, if $\xi=0$, the formula defining $\tilde{H}$ becomes a strict convolution (cf. Eq. (5)) and, as a matter of fact, in this case GPDs have a probabilistic interpretation, being the Fourier transforms of the impact factor dependent parton distributions [6]. Our formalism is therefore consistent with the fact that convolutions, in the probabilistic sense, arise once we limit the expressions to quantities having a probabilistic interpretation.

Let us now specify the formalism to the proton case and to CQM calculations. If the $E$-dependence of $\xi^{\prime}$, i.e., the $E$-dependence of $\tilde{z}$ (cf. Eq. (28)) is disregarded in Eq. (33), so that the spin dependent, off-diagonal momentum distribution

$$
\tilde{n}_{q_{0}}(\vec{p}, \vec{p}+\vec{\Delta})=\int d E \tilde{P}_{q_{0}}(\vec{p}, \vec{p}+\vec{\Delta}, E)
$$

is recovered, Eq.(33) can be written in the form

$$
\begin{aligned}
\tilde{H}_{q}\left(x, \xi, \Delta^{2}\right) & \simeq \sum_{q_{0}} \int d \vec{p} \tilde{n}_{q_{0}}(\vec{p}, \vec{p}+\vec{\Delta}) \frac{\xi^{\prime}}{\xi} \tilde{H}_{q_{0} q}\left(x^{\prime}, \xi^{\prime}, \Delta^{2}\right)= \\
& =\sum_{q_{0}} \int_{x}^{1} \frac{d z}{z} \int d \vec{p} \tilde{n}_{q_{0}}(\vec{p}, \vec{p}+\vec{\Delta}) \delta\left(z-\frac{\xi}{\xi^{\prime}}\right) \tilde{H}_{q_{0} q}\left(\frac{x}{z}, \frac{\xi}{z}, \Delta^{2}\right),
\end{aligned}
$$

which can also be cast in the form of Eq. (38), with the light-cone off-diagonal momentum distribution given by

$$
\tilde{H}_{q_{0}}\left(z, \xi, \Delta^{2}\right)=\int d \vec{p} \tilde{n}_{q_{0}}(\vec{p}, \vec{p}+\vec{\Delta}) \delta\left(z+\xi-\frac{p^{+}}{\bar{P}^{+}}\right)
$$


which coincides with Eq. (22) and it is to be evaluated in a given CQM, for $q_{0}=u_{0}$ or $d_{0}$, while $\tilde{H}_{q_{0} q}\left(\frac{x}{z}, \frac{\xi}{z}, \Delta^{2}\right)$ is the constituent quark helicity-dependent GPD, which is still to be discussed and will be modelled in the next section.

\section{A MODEL FOR THE HELICITY-DEPENDENT GPD OF THE CONSTITUENT QUARK}

The main issue remaining is the definition of $\tilde{H}_{q_{0} q}\left(\frac{x}{z}, \frac{\xi}{z}, \Delta^{2}\right)$, the helicity-dependent constituent quark GPD, appearing in Eq. (38).

As it has been done in Ref. [29] for the unpolarized case, this quantity will be modelled to respect that its forward limit, recovers the constituent quark parton distributions. We adopt the picture proposed in $[30,31]$ that the constituent quark is a complex system of point-like partons described by the scheme suggested by Altarelli, Cabibbo, Maiani and Petronzio (ACMP) [32]. In this scheme the constituent quarks are composite objects whose

structure functions are described by a set of functions $\phi_{q_{0} q}(x)$ that specify the number of point-like partons of type $q$ which are present in the constituent of type $q_{0}$, with fraction $x$ of the total momentum. We call these functions, generically, the structure functions of the constituent quark.

The functions describing the nucleon parton distributions are expressed in terms of the independent $\phi_{q_{0} q}(x)$ and of the constituent density distributions $\left(q_{0}=u_{0}, d_{0}\right)$ as,

$$
q\left(x, Q^{2}\right)=\sum_{q_{0}} \int_{x}^{1} \frac{d z}{z} q_{0}\left(z, Q^{2}\right) \phi_{q_{0} q}\left(\frac{x}{z}, Q^{2}\right)
$$

where $q$ labels the various partons, i.e., valence quarks $\left(u_{v}, d_{v}\right)$, sea quarks $\left(u_{s}, d_{s}, s\right)$, sea antiquarks $(\bar{u}, \bar{d}, \bar{s})$ and gluons $g$.

The different structure functions of the constituent quarks are derived from three very natural assumptions [32]: i) the point-like partons are $Q C D$ degrees of freedom, i.e. quarks, antiquarks and gluons; ii) Regge behavior for $x \rightarrow 0$ and duality ideas; iii) invariance under charge conjugation and isospin. 
These considerations define in the case of the valence quarks the following structure function

$$
\phi_{q q_{v}}\left(x, Q^{2}\right)=\frac{\Gamma\left(A+\frac{1}{2}\right)}{\Gamma\left(\frac{1}{2}\right) \Gamma(A)} \frac{(1-x)^{A-1}}{\sqrt{x}} .
$$

For the sea quarks the corresponding structure function becomes,

$$
\phi_{q q_{s}}\left(x, Q^{2}\right)=\frac{C}{x}(1-x)^{D-1}
$$

and, in the case of the gluons, it is taken

$$
\phi_{q g}\left(x, Q^{2}\right)=\frac{G}{x}(1-x)^{B-1}
$$

The last input of the approach consists of the definition of the scale at which the constituent quark structure is defined. We choose the so called hadronic scale $\mu_{0}^{2}[21,22]$. This last hypothesis fixes all the parameters of the approach (Eqs. (48) through (50)). The constants $A, B, G$ and the ratio $C / D$ are determined by the amount of momentum carried by the different partons, corresponding to a hadronic scale of $\mu_{0}^{2}=0.34 \mathrm{GeV}^{2}$, according to the parameterization of [21]. $C$ (or $D$ ) is fixed according to the value of $F_{2}$ at $x=0.01$ [32], and its value is chosen again according to [21]. We stress that all these inputs are forced only by the updated phenomenology, through the $2^{\text {nd }}$ moments of PDFs. The values of the parameters obtained are listed in [30].

The other ingredients appearing in Eq. (47), i.e., the density distributions for each constituent quark, represent the unpolarized equivalent of Eq. (22).

In Ref. [31], the spin dependent structure function of the constituent quark have been built generalizing the analysis of Ref. [30], leading to Eqs. (48) - (50). In here, we review the procedure addressed in Ref. [31]. Let

$$
\Delta q\left(x, \mu_{0}^{2}\right)=q_{+}\left(x, \mu_{0}^{2}\right)-q_{-}\left(x, \mu_{0}^{2}\right)
$$

where \pm label the quark spin projections and $q$ represents any flavor. The $A C M P$ approach, generalized to the polarized case, implies 


$$
q_{i}(x)=\sum_{q_{0}} \int_{x}^{1} \frac{d z}{z} \sum_{j} q_{0 j}(z) \Phi_{q_{0 j} q_{i}}\left(\frac{x}{z}\right)
$$

where $i= \pm$ labels the partonic spin projections, $j= \pm$ the constituent quark spins and $q_{0}=u_{0}, d_{0}$. Using spin symmetry we arrive at

$$
\left.\Delta q(x)=\int_{x}^{1} \frac{d z}{z}\left[\Delta u_{0}(z) \Delta \Phi_{u_{0} q}\left(\frac{x}{z}\right)+\Delta d_{0}(z) \Delta \Phi_{d_{0} q}\left(\frac{x}{z}\right)\right)\right]
$$

where $\Delta q_{0}=q_{0+}-q_{0-}$, and

$$
\Delta \Phi_{q_{0} q}=\Phi_{q_{0}+q+}-\Phi_{q_{0}+q-}
$$

Note at this point that the unpolarized case can be described in this generalized formalism as

$$
q(x)=q_{+}(x)+q_{-}(x)=\sum_{q_{0}} \int_{x}^{1} \frac{d z}{z} q_{0}(z) \Phi_{q_{0} q}\left(\frac{x}{z}\right)
$$

where

$$
\Phi_{q_{0} q}=\Phi_{q_{0}+q+}+\Phi_{q_{0}+q-}
$$

The description, reformulated in terms of the conventional valence and sea quark separation, yields

$$
\begin{gathered}
\Delta q(x)=\Delta q_{v}(x)+\Delta q_{s}(x)= \\
\sum_{q_{0}} \int_{x}^{1} \frac{d z}{z} \Delta q_{0}(z)\left[\Delta \Phi_{q_{0} q_{v}}\left(\frac{x}{z}\right)+\Delta \Phi_{q_{0} q_{s}}\left(\frac{x}{z}\right)\right]
\end{gathered}
$$

Since $\Delta \Phi_{q_{0} q_{v}}=\Delta \Phi_{q_{0} q_{v}} \delta_{q_{0} q}$, i.e. any constituent has a leading parton with the same quantum numbers, one has

$$
\begin{gathered}
\Delta q_{v}(x)=\int_{x}^{1} \frac{d z}{z} \Delta q_{0}(z) \Delta \Phi_{q_{0} q_{v}}\left(\frac{x}{z}\right) \\
\Delta q_{s}(x)=\sum_{q_{0}} \int_{x}^{1} \frac{d z}{z} \Delta q_{0}(z) \Delta \Phi_{q_{0} q_{s}}\left(\frac{x}{z}\right)
\end{gathered}
$$


Introducing $S U(6)$ (spin-isospin) symmetry as a simplifying assumption, gives

$$
\Delta \Phi_{u_{0} u}=\Delta \Phi_{d_{0} d}
$$

and

$$
\Delta \Phi_{u_{0} d}=\Delta \Phi_{d_{0} u}
$$

Furthermore they imply

$$
\Delta \Phi_{u_{0} u_{v}}+\Delta \Phi_{u_{0} u_{s}}=\Delta \Phi_{d_{0} d_{v}}+\Delta \Phi_{d_{0} d_{s}}
$$

and

$$
\Delta \Phi_{u_{0} d_{s}}=\Delta \Phi_{d_{0} u_{s}}
$$

By adding to these the following relations

$$
\begin{aligned}
& \Delta \Phi_{u_{0} u_{s}}=\Delta \Phi_{d_{0} u_{s}}, \\
& \Delta \Phi_{d_{0} d_{s}}=\Delta \Phi_{u_{0} d_{s}},
\end{aligned}
$$

which are beyond $S U(6)$ symmetry, but quite reasonable, one obtains,

$$
\Delta \Phi_{u_{0} u_{s}}=\Delta \Phi_{d_{0} u_{s}}=\Delta \Phi_{u_{0} d_{s}}=\Delta \Phi_{d_{0} d_{s}}=\Delta \Phi_{q_{0} q_{s}}
$$

and

$$
\Delta \Phi_{u_{0} u_{v}}=\Delta \Phi_{d_{0} d_{v}}=\Delta \Phi_{q_{0} q_{v}} .
$$

In this way the structure functions for the valence and for the sea are reduced to just two independent constituent structure functions and Eq. (59) simplifies to

$$
\Delta q_{s}(x)=\int_{x}^{1} \frac{d z}{z}\left(\Delta u_{0}(z)+\Delta d_{0}(z)\right) \Delta \Phi_{q_{0} q_{s}}\left(\frac{x}{z}\right) .
$$

The same argumentation applied to gluons implies 


$$
\Delta g(x)=\int_{x}^{1} \frac{d z}{z}\left(\Delta u_{0}(z)+\Delta d_{0}(z)\right) \Delta \Phi_{q_{0} g}\left(\frac{x}{z}\right)
$$

Thus the $A C M P$ procedure can be extended to the polarized case by introducing three additional structure functions for the constituent quarks: $\Delta \Phi_{q_{0} q_{v}}, \Delta \Phi_{q_{0} q_{s}}$ and $\Delta \Phi_{q_{0} g}$.

In order to determine the polarized constituent structure functions we add some assumptions which will determine them and those of the unpolarized case using a small number of parameters. They are: iv) factorization assumption: $\Delta \Phi$ cannot depend upon the quark model used, i.e, cannot depend upon the particular $\Delta q_{0} ; \mathrm{v}$ ) positivity assumption: the positivity constraint $\Delta \Phi \leq \Phi$ is saturated for $x=1$. These additional assumptions determine completely the parameters of the polarized constituent structure functions. In fact, the $Q C D$ partonic picture, Regge behavior and duality imply that

$$
\Delta \Phi_{q_{0} f}=\Delta C_{f} x^{a_{f}}(1-x)^{A_{f}-1}
$$

and $0<a_{f}<\frac{1}{2}$, for all $f=q_{v}, q_{s}, g$, as defined by dominant exchange of the $A_{1}$ meson trajectory [48].

The positivity restriction, $\Delta \Phi \leq \Phi$, is a natural consequence of the probability interpretation of the parton distributions. The assumption that the inequality is saturated for $x=1$, in the spirit of ref. [49], implies that $\Delta C_{f}=C_{f}$, the latter being the parameter fixed in the analysis of the unpolarized case, and therefore when $x \approx 1$ the partons which carry all of the momentum also carry all of the polarization, i.e., $\Phi_{+-}=0$. As is discussed in Ref. [31], this scheme has been changed only for the sea structure function, in order to obtain a reasonable description of the polarized sea. This led to the definition of a parameter, $\Delta C_{q_{s}}$, different from the one, $C_{q_{s}}$ defined in the unpolarized case. From the point of view of the number of parameters, this is a minimal assumption, since it reduces the parameters of the polarized case to those of the unpolarized one, with the only exception of $C_{q_{s}}$. Lastly the exponents $A_{f}$ are also taken from the unpolarized case. To summarize the parameterization, let us stress that the main change between the polarized functions and the unpolarized ones comes only from Regge behavior and the only parameter which has been changed is the one related to the polarized sea distribution. 
With all the above hypothesis the constituent quark functions become

$$
\begin{gathered}
\Delta \Phi_{q q_{v}}\left(x, \mu_{0}^{2}\right)=\frac{\Gamma\left(A+\frac{1}{2}\right)}{\Gamma\left(\frac{1}{2}\right) \Gamma(A)} x^{a_{f}}(1-x)^{A-1} \\
\Delta \Phi_{q q_{s}}\left(x, \mu_{0}^{2}\right)=\Delta C_{s} x^{a_{f}}(1-x)^{D-1} \\
\Delta \Phi_{q g}\left(x, \mu_{0}^{2}\right)=G x^{a_{f}}(1-x)^{B-1}
\end{gathered}
$$

where $A, D, B, G$ are the parameters of the unpolarized case, while $\Delta C_{s}$ is taken to reproduce the polarized data. In what follows, we shall use $0 \leq a_{f} \leq 0.5$, the range proposed in Ref. [50] in agreement with Ref. [48].

We have to generalize this scenario to describe off-forward phenomena. Of course, the forward limit of our GPDs formula, Eq. (38), has to be given by Eq. (57). By taking the forward limit of Eq. (38), one obtains:

$$
\begin{aligned}
\tilde{H}_{q}(x, 0,0) & =\sum_{q_{0}} \int_{x}^{1} \frac{d z}{z} \tilde{H}_{q_{0}}(z, 0,0) \tilde{H}_{q_{0} q}\left(\frac{x}{z}, 0,0\right) \\
& =\sum_{q_{0}} \int_{x}^{1} \frac{d z}{z} \Delta q_{0}(z) \tilde{H}_{q_{0} q}\left(\frac{x}{z}, 0,0\right),
\end{aligned}
$$

so that, in order for the latter to coincide with Eq. (57), one must have $\tilde{H}_{q_{0} q}(x, 0,0) \equiv$ $\Delta \phi_{q_{0} q}(x)$

In such a way, through the ACMP prescription, the forward limit of the unknown constituent quark GPD $\tilde{H}_{q_{0} q}\left(\frac{x}{z}, \frac{\xi}{z}, \Delta^{2}\right)$ can be fixed.

The off-forward behavior of the Constituent Quark GPDs has to be modelled and it will be done, as in Ref. [29] for the unpolarized case, using the " $\alpha$-Double Distributions" (DD's) language proposed by Radyushkin [36,37]. The DD's, $\Phi\left(\tilde{x}, \alpha, \Delta^{2}\right)$, are a representation of GPDs which automatically guarantees the polynomiality property. GPDs can be obtained from DD's after a proper integration, and, as is explained in [36,37], parton densities are recovered in the forward limit, while meson distribution amplitudes are obtained in the $P=0$ limit of the DD's. In some cases, such a transparent physical interpretation, together with 
the symmetry properties which are typical of distribution amplitudes ( $\alpha \rightarrow-\alpha$ symmetry), allows a direct modelling, already developed in [16].

The relation between any GPD $\tilde{H}$, defined à la $J i$, for example the one we need, i.e. $\tilde{H}_{q_{0} q}$ for the constituent quark target, is related to the $\alpha$-DD's, which we call $\Delta \tilde{\Phi}_{q_{0} q}\left(\tilde{x}, \alpha, \Delta^{2}\right)$ for the constituent quark, in the following way $[36,37]$ :

$$
\tilde{H}_{q_{0} q}\left(x, \xi, \Delta^{2}\right)=\int_{-1}^{1} d \tilde{x} \int_{-1+|\tilde{x}|}^{1-|\tilde{x}|} \delta(\tilde{x}+\xi \alpha-x) \Delta \tilde{\Phi}_{q_{0} q}\left(\tilde{x}, \alpha, \Delta^{2}\right) d \alpha
$$

With some care, the expression above can be integrated over $\tilde{x}$ and the result is explicitly given in [37], where a factorized ansatz is suggested for the DD's which, for the helicitydependent one, could be written:

$$
\Delta \tilde{\Phi}_{q_{0} q}\left(\tilde{x}, \alpha, \Delta^{2}\right)=h(\tilde{x}, \alpha) \Delta \Phi_{q_{0} q}(\tilde{x}) G_{q_{0}}^{q}\left(\Delta^{2}\right)
$$

with the $\alpha$ dependent term, $h(\tilde{x}, \alpha)$, having the character of a mesonic amplitude.

Besides, in Eq. (76) $\Delta \Phi_{q_{0} q}(\tilde{x})$ represents the forward density and, eventually, $G_{q_{0}}\left(\Delta^{2}\right)$ the constituent quark axial ff.

One immediately realizes that the helicity-dependent GPD of the constituent quark, Eq. (75), with the factorized form Eq. (76), fulfills the crucial constraints of GPDs, i.e., the forward limit, the first-moment and the polynomiality condition, the latter being automatically verified in the DD's description.

In the following, it will be assumed for the constituent quark GPD the above factorized form, so that one needs to model the three functions appearing in Eq. (76), according to the present description.

For the amplitude $h$, use will be made of one of the simple normalized forms suggested in [36], on the bases of the symmetry properties of DD's:

$$
h(\tilde{x}, \alpha)=\frac{3}{4} \frac{(1-\tilde{x})^{2}-\alpha^{2}}{(1-\tilde{x})^{3}} .
$$

In addition, since we will identify quarks for $x \geq \xi / 2$, pairs for $x \leq|\xi / 2|$, antiquarks for $x \leq-\xi / 2$, and, since in our approach the forward densities $\Delta \Phi_{q_{0} q}(\tilde{x})$ have to be given by 
the standard $\triangle \Phi$ functions of the $A C M P$ approach, Eqs. (71)-(73), one has, for the DD of flavor $q$ of the constituent quark:

$$
\Delta \tilde{\Phi}_{q_{0} q}\left(\tilde{x}, \alpha, \Delta^{2}\right)= \begin{cases}\left(h(\tilde{x}, \alpha) \tilde{\Phi}_{q_{0} q_{v}}(\tilde{x})+h(\tilde{x}, \alpha) \tilde{\Phi}_{q_{0} q_{s}}(\tilde{x})\right) G_{q_{0}}^{q}\left(\Delta^{2}\right) & \text { for } \tilde{x} \geq 0 \\ -h(-\tilde{x}, \alpha) \tilde{\Phi}_{q_{0} q_{s}}(-\tilde{x}) G_{q_{0}}^{q}\left(\Delta^{2}\right) & \text { for } \tilde{x}<0\end{cases}
$$

The above definition, when integrated over $\alpha$ gives the correct limits [37]:

$$
\left.\int_{-1+\tilde{x}}^{1-\tilde{x}} \Delta \tilde{\Phi}_{q_{0} q}\left(\tilde{x}, \alpha, \Delta^{2}=0\right)\right|_{\tilde{x}>0} d \alpha=\Delta \Phi_{q_{0} q}(\tilde{x})
$$

and

$$
\left.\int_{-1+|\tilde{x}|}^{1-|\tilde{x}|} \Delta \tilde{\Phi}_{q_{0} q}\left(\tilde{x}, \alpha, \Delta^{2}=0\right)\right|_{\tilde{x}<0} d \alpha=-\Delta \Phi_{q_{0} \bar{q}}(-\tilde{x})
$$

Eventually, as axial ff we will take a simple monopole form corresponding to a constituent quark size $r_{Q} \simeq 0.3 \mathrm{fm}$ :

$$
G_{q_{0}}\left(\Delta^{2}\right)=\frac{1}{1-\frac{\Delta^{2} r_{Q}^{2}}{6}}
$$

showing i.e. the same $Q^{2}$ dependence of the electromagnetic ff used in [29], the latter scenario being strongly supported by the analysis of [33].

By using such a ff and Eq. (77), together with the standard ACMP $\Delta \Phi$ 's, Eqs. (71) (73), in Eq. (78), and inserting the obtained $\Delta \Phi_{q_{0} q}\left(\tilde{x}, \alpha, \Delta^{2}\right)$ into Eq. (75), the constituent quark GPD in the ACMP scenario can be eventually calculated.

Let us discuss our factorized choice for the DD of the constituent quark, Eq. (76). Such a factorization does not hold for the GPD of the proton [4]. No microscopic calculation supports it [23]. The present approach is consistent with the absence of factorization of the proton GPD in $\Delta^{2}$-dependent and $\Delta^{2}$-independent terms since an additional, crucial, $\Delta^{2}$ dependence comes from the quantity $H_{q_{0}}$ in Eq. (38), i.e., from the proton wave function of the quark model used.

The factorized form is assumed for the GPD of the constituent quark for simplicity. We do not know what the helicity-dependent GPD of the constituent quark is and we start modelling it by using the simplest possible structure which fulfills the required constraints. 
Clearly this choice deserves a deeper explanation, which we do not have at the moment. However, one expects that the structure of the constituent quark GPD is closer to the asymptotic behavior, than that of the proton, because it probes higher energies. The non perturbative contributions arise in the precise shape of the three functions building the GPD, which we take from general QCD based arguments and from data. It is true that even the forward structure of the constituent quark is not known, but we use a parameterization which performs well in describing the data [31]. In this sense, the forward limit is well reproduced, as shown in the next section. On the contrary, the profile Eq. (77) is assumed to be valid also for the constituent quark, even in the helicity dependent case, simply because it satisfies some of the required properties.

These arguments and a small set of parameters, fixed once forever, make our approach able, if a proper CQM is used, to describe DIS data for parton distributions, elastic form factors, and to predict unpolarized and polarized GPDs. It allows for an effective description of the known structure of the nucleon and its predictive power needs to be tested with new observables.

\section{RESULTS AND DISCUSSION}

In this section we present the results obtained for the helicity-dependent GPD $\tilde{H}_{q}$ of the proton, for $\xi^{2} \ll 1$ and $\vec{\Delta}^{2} \ll m^{2}$, according to the approach described so far. The main equation to be evaluated is Eq. (38), written again here below for the sake of clarity:

$$
\tilde{H}_{q}\left(x, \xi, \Delta^{2}\right)=\sum_{q_{0}} \int_{x}^{1} \frac{d z}{z} \tilde{H}_{q_{0}}\left(z, \xi, \Delta^{2}\right) \tilde{H}_{q_{0} q}\left(\frac{x}{z}, \frac{\xi}{z}, \Delta^{2}\right) .
$$

In the above equation, the quantity $\tilde{H}_{q_{0} q}$, the helicity-dependent constituent quark GPD, is modelled according to the arguments described in the previous section. This means that it is obtained evaluating Eq. (75), where the DD of the constituent quark, $\Delta \tilde{\Phi}_{q_{0} q}\left(\tilde{x}, \alpha, \Delta^{2}\right)$ , is given by Eq. (78), calculated in turn through the ff, Eq. (81), the function $h$, Eq. (77), together with the forward ACMP $\Delta \Phi$ 's, Eqs. (71) - (73). 
The other ingredient in Eq. (38), $\tilde{H}_{q_{0}}$, has been evaluated according to Eq. (22):

$$
\tilde{H}_{q_{0}}\left(z, \xi, \Delta^{2}\right)=\int d \vec{p} \tilde{n}_{q_{0}}(\vec{p}, \vec{p}+\vec{\Delta}) \delta\left(z+\xi-\frac{p^{+}}{\bar{P}^{+}}\right) .
$$

The calculation has been performed in the Breit frame, where one has, in the NR limit studied, $\sqrt{2} \bar{P}^{+} \rightarrow M$. The off-diagonal momentum distribution appearing in the formula above, $\tilde{n}_{q_{0}}(\vec{p}, \vec{p}+\vec{\Delta})$, defined in Eq. (44), has been evaluated within the Isgur and Karl (IK) model [24], according to the definition

$$
\tilde{n}_{q_{0}}(\vec{p}, \vec{p}+\vec{\Delta})=\tilde{n}_{q_{0}+}(\vec{p}, \vec{p}+\vec{\Delta})-\tilde{n}_{q_{0}-}(\vec{p}, \vec{p}+\vec{\Delta})
$$

where $n_{q_{0 \pm}}(\vec{p}, \vec{p}+\vec{\Delta})$ is the off-diagonal momentum distribution for the valence quark momentum $\vec{p}$ and spin projection parallel or antiparallel to that of the nucleon. $n_{q \pm}(\vec{p}, \vec{p}+\vec{\Delta})$ can be evaluated projecting out the appropriate spin and flavor component of the constituent quark and in the corresponding baryonic state is given by

$$
\begin{aligned}
\tilde{n}_{u_{0} / d_{0}, \pm}(\vec{p}, \vec{p}+\vec{\Delta}) & =3\left({ }_{s f}<N, J_{z}=+\frac{1}{2}\left|\frac{1 \pm \tau_{3}^{z}}{2} \frac{1 \pm \sigma_{3}^{z}}{2}\right| N, J_{z}=+\frac{1}{2}>_{s f}\right) \times \\
& \times \int d \vec{p}_{1} d \vec{p}_{2} \psi^{*}\left(\vec{p}_{1}, \vec{p}_{2}, \vec{p}+\vec{\Delta}\right) \psi\left(\vec{p}_{1}, \vec{p}_{2}, \vec{p}\right)
\end{aligned}
$$

where $\mid N, J_{z}=+\frac{1}{2}>_{s f}$ is the spin-flavor state and $\psi\left(\vec{p}_{1}, \vec{p}_{2}, \vec{p}_{3}\right)$ is the proton wave function in momentum space (cf. Ref. [23] for the definition in the unpolarized case).

In the IK CQM [24], including contributions up to the $2 \hbar \omega$ shell, the proton state is given by the following admixture of states

$$
\left.\left.\left.\left.|N\rangle=\left.a_{\mathcal{S}}\right|^{2} S_{1 / 2}\right\rangle_{S}+\left.a_{\mathcal{S}^{\prime}}\right|^{2} S_{1 / 2}^{\prime}\right\rangle_{S}+\left.a_{\mathcal{M}}\right|^{2} S_{1 / 2}\right\rangle_{M}+\left.a_{\mathcal{D}}\right|^{4} D_{1 / 2}\right\rangle_{M}
$$

where the spectroscopic notation $\left.\left.\right|^{2 S+1} X_{J}\right\rangle_{t}$, with $t=A, M, S$ being the symmetry type, has been used. The coefficients were determined by spectroscopic properties to be [51]: $a_{\mathcal{S}}=0.931, a_{\mathcal{S}^{\prime}}=-0.274, a_{\mathcal{M}}=-0.233, a_{\mathcal{D}}=-0.067$.

The results for the GPD $\tilde{H}\left(x, \xi, \Delta^{2}\right)$, neglecting in (84) the small $D$-wave contribution, are found to be: 


$$
\begin{aligned}
\tilde{H}_{u}\left(x, \xi, \Delta^{2}\right) & =3 \frac{M}{\alpha^{3}}\left(\frac{3}{2 \pi}\right)^{3 / 2} e^{\frac{\Delta^{2}}{3 \alpha^{2}}} \int d k_{x} \int d k_{y} f_{0}\left(k_{x}, k_{y}, x, \xi, \Delta^{2}\right) \\
& \times\left[\Delta f_{s}\left(k_{x}, k_{y}, x, \xi, \Delta^{2}\right)+\Delta \tilde{f}\left(k_{x}, k_{y}, x, \xi, \Delta^{2}\right)\right], \\
\tilde{H}_{d}\left(x, \xi, \Delta^{2}\right) & =3 \frac{M}{\alpha^{3}}\left(\frac{3}{2 \pi}\right)^{3 / 2} e^{\frac{\Delta^{2}}{3 \alpha^{2}}} \int d k_{x} \int d k_{y} f_{0}\left(k_{x}, k_{y}, x, \xi, \Delta^{2}\right) \\
& \times\left[-\frac{1}{4} \Delta f_{s}\left(k_{x}, k_{y}, x, \xi, \Delta^{2}\right)+\frac{1}{5} \Delta \tilde{f}\left(k_{x}, k_{y}, x, \xi, \Delta^{2}\right)+\bar{f}\left(k_{x}, k_{y}, x, \xi, \Delta^{2}\right)\right],
\end{aligned}
$$

for the flavors $u$ and $d$, respectively, with

$$
\begin{aligned}
& f_{0}\left(k_{x}, k_{y}, x, \xi, \Delta^{2}\right)=\frac{\bar{k}_{0}}{\bar{k}_{0}+\bar{k}_{z}} f_{\alpha}\left(\Delta_{x}, k_{x}\right) f_{\alpha}\left(\Delta_{y}, k_{y}\right) f_{\alpha}\left(\Delta_{z}, \bar{k}_{z}\right) \\
& f_{\alpha}\left(\Delta_{i}, k_{i}\right)=e^{-\frac{1}{\alpha^{2}}\left(\frac{3}{2} k_{i}^{2}+k_{i} \Delta_{i}\right)} \\
& \bar{k}_{z}=\frac{M^{2}(x+\xi)^{2}-\left(m^{2}+k_{x}^{2}+k_{y}^{2}\right)}{2 M(x+\xi)}, \\
& \Delta f_{s}\left(k_{x}, k_{y}, x, \xi, \Delta^{2}\right)=\frac{4}{9} a_{s}^{2}+\frac{4}{9} a_{s^{\prime}}^{2}\left[\frac{5}{4}-\frac{3}{2} \frac{k^{2}}{\alpha^{2}}+\frac{3}{4} \frac{k^{4}}{\alpha^{4}}+\frac{1}{\alpha^{2}}\left(-\frac{\Delta^{2}}{3}+\vec{\Delta} \cdot \vec{k}\right)\left(\frac{k^{2}}{\alpha^{2}}-1\right)\right] \\
& +\frac{4}{9} a_{M}^{2}\left[\frac{5}{8}-\frac{3}{4} \frac{k^{2}}{\alpha^{2}}+\frac{3}{8} \frac{k^{4}}{\alpha^{4}} \frac{1}{2 \alpha^{2}}\left(-\frac{\Delta^{2}}{3}+\vec{\Delta} \cdot \vec{k}\right)\left(\frac{k^{2}}{\alpha^{2}}-1\right)\right] \\
& -\frac{4}{9} \sqrt{3} a_{S} a_{S^{\prime}}\left[\left(-1+\frac{k^{2}}{\alpha^{2}}\right)-\frac{2}{3 \alpha^{2}}\left(\frac{\Delta^{2}}{3}-\vec{\Delta} \cdot \vec{k}\right)\right] \\
& \Delta \tilde{f}\left(k_{x}, k_{y}, x, \xi, \Delta^{2}\right)=-\frac{1}{\sqrt{2}} \frac{5}{3} a_{M} a_{S^{\prime}}\left[\frac{1}{6}-\frac{k^{2}}{\alpha^{2}}+\frac{1}{2} \frac{k^{4}}{\alpha^{4}}-\frac{2}{3 \alpha^{2}}\left(-\frac{\Delta^{2}}{3}-\vec{\Delta} \cdot \vec{k}\right)\right. \\
& \left.+\frac{2 k^{2}}{3 \alpha^{4}}\left(-\frac{\Delta^{2}}{3}+\vec{\Delta} \cdot \vec{k}\right)\right] \\
& \bar{f}\left(k_{x}, k_{y}, x, \xi, \Delta^{2}\right)=\frac{1}{9} \frac{k}{\alpha^{2}} \sqrt{\frac{9}{4} k^{2}-\Delta^{2}+3 \vec{\Delta} \cdot \vec{k}},
\end{aligned}
$$

and $\bar{k}_{0}=\sqrt{m^{2}+k_{x}^{2}+k_{y}^{2}+\bar{k}_{z}^{2}}$, being $m \simeq M / 3$ the constituent quark mass. Here we have used the notation $k^{2}=\vec{k}^{2}, \vec{k}=\left(k_{x}, k_{y}, k_{z}\right)$. Evaluating the forward limit, $\Delta^{2}=0, \xi=0$, of Eqs. (85) and (86), the expressions of the polarized quark densities in the IK model, obtained in [41], are consistently recovered. 
The harmonic oscillator parameter, $\alpha$, of the IK model, has ben chosen so that the experimental r.m.s. of the proton is reproduced by the slope, at $\Delta^{2}=0$, of the charge ff, so that a value of $\alpha=1.18 f^{-1}$ is obtained [29]. Such a ff reproduces well the data at the low values of $\Delta^{2}$ which are accessible in the present approach. For higher values of $\Delta^{2}$, it would not be realistic [51]. The same quality of results is expected for the proton axial form factor, which is obtained by integrating Eq. (38) over $x$, according to Eq. (42). The experimental dipole trend [52] is qualitatively recovered at the low values of $\Delta^{2}$ under scrutiny here.

Results for the $u$-quark $\tilde{H}_{u}$ distribution, at the scale of the model $\mu_{o}^{2}$, are shown in Figs 2 to 4 . In Fig. 2, it is shown for $\Delta^{2}=-0.1 \mathrm{GeV}^{2}$ and $\xi=0.1$. One should remember that the present approach does not allow to estimate realistically the region $-\Delta^{2} \geq m^{2} \simeq 0.1$ $\mathrm{GeV}^{2}$, so that we are showing here the result corresponding to the highest possible $\Delta^{2}$ value. Accordingly, the maximum value of the skewedness is therefore $\xi \simeq 0.17$ (cf. Eq. (2)), fulfilling the requirement $\xi^{2} \ll 1$. The dashed curve represents what is obtained in the pure IK model, i.e., by evaluating Eq. (85). One should notice that such a result, obtained in a pure valence CQM, should vanish for $x \leq \xi$ and for $x \geq 1$. The small tails which are found in these forbidden regions represent the amount of support violation of the approach. In particular, for the shown values of $\Delta^{2}$ and $\xi$, a violation of $2 \%$ is found. In general, in the accessible region the violation is never larger than few percents. The full curves in Fig. 3 represents the complete result of the present approach, i.e., the evaluation of Eq. (38) following the steps and using the ingredients described in this section and in the previous one. The two curves are obtained in correspondence of the two extreme values of the Regge intercept [48], $a_{f}=0$ (upper curve) and $a_{f}=0.5$ (lower curve), used to define the helicity dependent constituent quark structure functions [31]. A relevant contribution is found to lie in the ERBL region, in agreement with other estimates [10]. As already explained, the knowledge of GPDs in the ERBL region is a crucial prerequisite for the calculation of all the cross-sections and the observables measured in the processes where GPDs contribute. We notice that the ERBL region is accessed here, with respect to the approach of Ref. [23] generalized to the helicity-dependent case, which gives the dashed curve, thanks to the 
constituent structure which has been introduced implementing the ACMP procedure. A similar result has been found in [29] in the unpolarized case.

In Fig. 3, special emphasis is devoted to show the $\xi$-dependence of the results. For the allowed $\xi$ values, $\tilde{H}_{u}\left(x, \xi, \Delta^{2}\right)$, evaluated using our main formula, Eq. (38), is shown for four different values of $x$. It is clearly seen that such a dependence is stronger in the ERBL region, than in the DGLAP region, in good agreement with other estimates [10] and with our previous analysis, performed in the unpolarized case [29]. To allow for a complete view of the outcome of our approach, in Fig. 4 the $x$ and $\xi$ dependences are shown together in a 3-dimensional plot.

The results shown so far are associated with the low scale of the model, the hadronic scale $\mu_{0}^{2}$, fixed to the value $0.34 \mathrm{GeV}^{2}$, as discussed in Section 4 .

In order to have a feeling of the quality of the present predictions, since no data are available at the moment for $\tilde{H}\left(x, \xi, \Delta^{2}\right)$, we evaluate in the following the forward limit of the obtained quantities. In Figs. 5 and 6, the proton and neutron spin dependent structure function $g_{1}\left(x, Q^{2}\right)$, built from the forward limit of Eq. (38) evaluated for $q=$ $q_{v}, q_{s}, g$, are compared with experimental data. In Ref. [31], the succesful analysis had been performed using the model of Ref. [53], so that the encouraging results shown there have to be confirmed when the IK model is considered, as it is being done here. To evaluate $g_{1}\left(x, Q^{2}\right)$ at the experimental scale, a NLO evolution of the model parton distributions has been performed. It is known that perturbative $Q C D$ to this order allows the proposal of varied factorization schemes [54], whereby one is able to define the partonic distributions in different ways without altering the physical observables. In our analysis the parton distributions are determined by the quark model through the ACMP procedure. It is evident that different factorization schemes lead to different results. Following Ref. [31], we have adopted on physical grounds the $A B$ scheme as defined in ref. [55] ${ }^{1}$. In it some relevant physical

\footnotetext{
${ }^{1}$ It consists in modifying minimally the NLO polarized anomalous dimensions [56], calculated in
} 
observables, such as $\Delta \Sigma$, the total spin carried by the quarks, are scale independent, i.e., they behave as conserved quantities, and therefore the partons have a well defined interpretation in terms of constituents. Fig. 5 and 6 show that the present approach describes well the data $[60,61]$ for the proton structure function $g_{1}$, and the trend of the neutron data [62] is also reasonably reproduced. The quality of the agreement is comparable with that obtained in Ref. [31] using the model of [53].

The present approach in the forward limit, i.e. the description given in Ref. [31], also allows for the predictions of axial charges of the proton. By using the IK model, the following values are obtained (the first listed value corresponds to the Regge slope $a_{f}=0.5$, the second to $a_{f}=0$. ): for the octet charge, $a_{8}=0.540 \div 0.671$ (experimental value: $0.58 \pm 0.03$ [58]), for the isospin charge, $a_{3}=0.857 \div 1.063$ (experimental value: $1.257 \pm 0.003$ [59]), while the predictions for the singlet charge and for the spin carried by the gluons lead to $\Delta \Sigma=0.402 \div 0.530$ (from the analysis of Ref. [50] : $0.45 \pm 0.09$ ). Again, the agreement with data of these results, obtained by using the IK wave functions, is comparable to the one obtained in Ref. [31] by using the wave functions of Ref. [53].

This finding deserves a further comment. The lack of high momentum components in the IK wave function with respect to that of the model of Ref. [53] would indicate the latter as a better tool to describe the data. Nevertheless, the low- $x$ behavior of the model results turns out to be mainly governed by the constituent quark structure rather than by the constituent quark wave function. The correct Regge-behavior given by our parametrization of the constituent quark structure functions provides us with a model independent prediction. Such a feature has not to do with the use of a relativistic model, which would improve only the high $x$ behavior. As discussed in Ref [63], relativity and constituent quark structure are independent features, both of them necessary if model calculations are used for a proper

the $\overline{M S}$ scheme, in order to have the axial anomaly governing the first moment of $g_{1}$, as proposed in Ref [57]. 
description of data in the full $x$ range.

Since no data are presently available for helicity-dependent GPDs, it is certainly useful to compare the results of model calculations with model independent constraints, if available. It has been proposed that they could be obtained from analysis of data in particular kinematical conditions. Very recently, this issue has been addressed in two papers, where GPDs have been obtained, at $\xi=0$, by fitting the first moment of a reasonable ansatz of GPDs to ff data $[64,65]$. Anyway, such a procedure is difficult to be realized for the quantity under scrutiny here, due to the lack of a satisfactory experimental knowledge of the axial ff, which cannot provide us with a clear constraint on $\tilde{H}_{q}\left(x, 0, \Delta^{2}\right)$. As a matter of fact, in Ref. [65] no prediction has been produced for it, so that no comparison is possible between the present results and those of [65]. On the contrary, some curves reproducing $\tilde{H}_{q}\left(x, 0, \Delta^{2}\right)$ have been shown in [64]. Nevertheless, as the authors of that paper clearly state, the lack of precise data for the axial ff does not allow a realistic parameterization of $\tilde{H}_{q}\left(x, 0, \Delta^{2}\right)$, but rather a simple ansatz for it. This ansatz is given by the product of the polarized parton distributions, taken from the "scenario 1" of Ref. [66] at $Q^{2}=4 \mathrm{GeV}^{2}$, and an exponential term, giving the $\Delta^{2}$ dependence and additional $x$ dependence, taking into account the different Regge behavior at different values of the momentum transfer. The latter dependence is borrowed from the analysis performed for the helicity independent GPDs, giving the so called default fit of [64], and no argument is given to support this procedure in the helicity dependent case. Anyway, we show in Fig. 7 the comparison of our results for the valence distribution $\tilde{H}_{u}^{v}\left(x, 0, \Delta^{2}\right)$, evaluated at $\Delta^{2}=-0.1 \mathrm{GeV}^{2}$, evolved to NLO from the hadronic scale up to $Q^{2}=4 \mathrm{GeV}^{2}$, with the ansatz proposed in [64], evaluated in the same kinematical conditions. It is seen that the latter and the present model predict a similar scenario. The very low $x$ behavior is governed in both cases by the Regge phenomenology, which is left here free to vary between the two full lines of the figure, while it is fixed in Ref. [64] in the way described above, producing a big enhancement at low values of $-\Delta^{2}$.

Summarizing this section, we have developed here a scheme which provides us with the helicity-dependent GPD $\tilde{H}$ in the full $x$ range. This is obtained thanks to the constituent 
quark structure, implemented dressing the three quarks of a CQM, where initially only the DGLAP region of GPDs was accessible. This is an important development, a prerequisite for any attempt to calculate cross sections and asymmetries of related processes. The next step of our studies will be indeed to use the obtained GPDs for the evaluation of cross sections.

\section{CONCLUSIONS}

A thorough analysis of both polarized and unpolarized data have shown, in previous work, that constituent quarks cannot be considered elementary when studied with highenergy probes. Certainly evolution is needed, i.e. the constituent quarks at the hadronic scale have to be undressed by incorporating bremsstrahlung in order to reach the Bjorken regime. Besides, the constituent quarks should be endowed with soft structure in order to approach the data. Thus, the constituent quarks, when under scrutiny by high-energy probes, seem to behave as complex systems, with a very different behavior from the current quarks of the basic theory. These features, which we found in structure functions, have also been recently discussed in form factors [33].

The formalism of composite constituent quarks has been recently applied to the study of generalized parton distributions, developing a formalism which expresses the hadronic GPDs in terms of constituent quarks GPDs by means of appropriate convolutions [29]. The approach has been extended here to treat helicity-dependent GPDs. To our knowledge, this represents the first calculation which gives, in a constituent quark framework, estimates of helicity-dependent GPDs in the ERBL region. Following the spirit of Ref. [29], this is done incorporating phenomenological features of various kinematical regimes. In particular, use has been made of the Radyushkin's factorization ansatz, thus our constituent quark GPDs are defined in terms of the product of three functions: i) the constituent quark polarized structure function, where we use the parameterization of [31] following the ACMP proposal [32]; ii) Radyushkin's double distributions [36]; iii) constituent quark form factor as suggested in Ref. [33]. Once the helicity-dependent GPDs are defined in this way, we 
have developed the scheme to incorporate them into any nucleon model by appropriate convolution. In order to show the type of predictions to which our proposal leads, we have used here, as an illustration, the IK [24] model. However, in this latest step of our scheme, any non-relativistic (or relativized) model can be used to define the hadronic GPDs.

As in the unpolarized case [29], the present scheme transforms a hadronic model, in whose original description only valence quarks appear, into one containing all kinds of partons (i.e., quarks, antiquarks and gluons). Moreover, the starting model produces no structure in the ERBL region, while after the structure of the constituent quark has been incorporated, it does. The completeness of the $x$-range, for the allowed $\Delta^{2}$ and $\xi\left(\Delta^{2}<<m^{2}\right.$ and $\xi^{2} \ll 1$ ), of the present description, is a prerequisite for the calculation of cross-sections and other observables in a wide kinematical range. Relativistic corrections, which permit to access a wider kinematical region, could be included in the approach.

We have reminded the reader of the calculation for the forward polarized structure functions to see how in this case, where experimental data are available, our scheme leads, even with a naive quark model, to a reasonable description of the data. Thereafter, we have proceeded to calculate the GPDs of physical interest to guide the preparation and analysis of future experiments. Our calculation seems to be consistent with an ansatz recently suggested in Ref. [64].

This work is the continuation of an attempt to describe the properties of hadrons in different kinematical and dynamical scenarios. Although our description can never be a substitute of Quantum Chromodynamics, it may serve to guide experimenters to physical processes where the theory might show interesting features, worthy of a more fundamental effort.

\section{ACKNOWLEDGMENTS}

Useful comments by B. Pire are gratefully acknowledged. S.S. thanks the Department of Theoretical Physics of the Valencia University, where part of this work has been done, 
for a warm hospitality and financial support. This work is supported in part by GVGRUPOS03/094, MCYT-FIS2004-05616-C02-01 and by the Italian MIUR through the PRIN Theoretical Studies of the Nucleus and the Many Body Systems. 


\section{REFERENCES}

[1] D. Müller, D. Robaschik, B. Geyer, F.M. Dittes, and J. Hořejši, Fortsch. Phys. 42, 101 (1994); hep-ph/9812448.

[2] A. Radyushkin, Phys. Lett. B 385, 333 (1996); Phys. Rev. D 56, 5524 (1997).

[3] X. Ji, Phys. Rev. Lett. 78, 610 (1997); Phys. Rev. D 55, 7114 (1997).

[4] M. Diehl, Phys. Rept. 388, 41 (2003).

[5] R.L. Jaffe and A.V. Manohar, Nucl. Phys. B 337, 509 (1990).

[6] M. Burkardt, Phys. Rev. D 62, 071503 (2000); Int. J. Mod. Phys. A 18, 173 (2003);

M. Diehl, Eur. Phys. J. C 25, 223 (2002); J.P. Ralston and B. Pire, Phys. Rev. D 66, 111501 (2002); A.V. Belitsky and D. Müller, Nucl. Phys. A711, 118 (2002); X. Ji, Phys. Rev. Lett. 91, 062001 (2003).

[7] B.A. Mecking, Nucl. Phys. A711, 330c (2002); K. Rith, Nucl. Phys. A711, 336c (2002); D. von Harrach, Nucl. Phys. A711, 342c (2002).

[8] P.A. Guichon and M. Vanderhaeghen, Prog. Part. Nuc. Phys. 41, 125 (1998).

[9] X. Ji, W. Melnitchouk, and X. Song, Phys. Rev. D 56, 5511 (1997); I.V. Anikin, D. Binosi, R. Medrano, S. Noguera, and V. Vento, Eur. Phys. J. A 14, 95 (2002).

[10] K. Goeke, M.V. Polyakov, and M. Vanderhaeghen, Prog. Part. Nucl. Phys. 47, 401 (2001).

[11] V.Yu. Petrov, P.V. Pobylitsa, M.V. Polyakov, I. Bornig, K. Goeke, and C. Weiss, Phys. Rev. D 57, 4325 (1998); M. Penttinen, M.V. Polyakov, and K. Goeke, Phys. Rev. D 62, $014024(2000)$.

[12] B.C. Tiburzi and G.A. Miller, Phys. Rev. C 64, 065204 (2001); Phys. Rev. D 65, 074009 (2002). 
[13] A. Mukherjee and M. Vanderhaeghen, Phys. Rev. D 67, 085020 (2003).

[14] L. Theussl, S. Noguera, and V. Vento, Eur.Phys.J.A20, 483 (2004).

[15] A. Freund and V. Guzey, Phys. Lett. B 462, 178 (1999); L. Frankfurt, A. Freund, V. Guzey, and M. Strikman, Phys. Lett. B 418, 345c (1998).

[16] A.V. Radyushkin, Phys. Lett. B 449, 81 (1999); I.V. Musatov and A.V. Radyushkin, Phys. Rev. D 61, 074027 (2000).

[17] A.V. Belitsky, D. Müller, L. Niedermeier, and A. Schäfer Phys. Lett. B 474, 163 (2000), and references therein; A. Freund and M.F. McDermott, Eur. Phys. J. C 23, 651 (2002), and references therein.

[18] A.V. Belitsky and D. Müller, Nucl. Phys. B589, 611 (2000); N. Kivel, Maxim V. Polyakov, A. Schäfer, and O.V. Teryaev, Phys. Lett. B 497, 73 (2001); A.V. Belitsky, A. Kirchner, D. Müller, and A. Schäfer, Phys. Lett. B 510, 117 (2001).

[19] D.Yu. Ivanov, B. Pire, L. Szymanowski, and O. Teryaev, Phys. Lett. B 550, 65 (2002).

[20] G. Parisi and R. Petronzio, Phys. Lett. B 62, 331 (1976); R.L. Jaffe and G.G. Ross, Phys. Lett. B 93, 313 (1980).

[21] M. Glück, E. Reya, and A. Vogt, Eur. Phys. J. C 5, 461 (1998) and references therein.

[22] M. Traini, V. Vento, A. Mair, and A. Zambarda, Nucl. Phys. A614, 472 (1997).

[23] S. Scopetta and V. Vento, Eur. Phys. J. A 16, 527 (2003).

[24] N. Isgur and G. Karl, Phys. Rev. D 18, 4187 (1978); Phys. Rev. D 19, 2653 (1979).

[25] S. Boffi, B. Pasquini, and M. Traini, Nucl. Phys. B649, 243 (2003).

[26] M. Diehl, T. Feldmann, R. Jakob, and P. Kroll, Nucl. Phys. B596, 33 (2001); Eur. Phys. J. C 8, 409 (1999).

[27] P. Faccioli, M. Traini, and V. Vento, Nucl. Phys. A656, 400 (1999). 
[28] S. Boffi, B. Pasquini, and M. Traini, Nucl. Phys. B680, 147 (2004).

[29] S. Scopetta and V. Vento, Phys. Rev. D 69, 094004 (2004).

[30] S. Scopetta, V. Vento, and M. Traini, Phys. Lett. B 421, 64 (1998).

[31] S. Scopetta, V. Vento, and M. Traini, Phys. Lett. B 442, 28 (1998).

[32] G. Altarelli, N. Cabibbo, L. Maiani, and R. Petronzio, Nucl. Phys. B69, 531 (1974); G. Altarelli, S. Petrarca, and F. Rapuano, Phys. Lett. B 373, 200 (1996).

[33] R. Petronzio, S. Simula, and G. Ricco, Phys. Rev. D 67, 094004 (2003).

[34] G. Morpurgo, Physics 2, 95 (1965).

[35] R.C. Hwa, Phys. Rev. D 22, 759 (1980); R.C. Hwa and C.B. Yang, Phys. Rev. C 66, 025204 (2002) and references therein.

[36] A.V. Radyushkin, Phys. Rev. D 59, 014030 (1999).

[37] A.V. Radyushkin, in M. Shifman, (Editor): At the Frontier of Particle Physics, Vol. 2, (World Scientific, Singapore, 2001) pp. 1037-1099, hep-ph/0101225;

[38] X. Ji, J. Phys. G 24, 1181 (1998).

[39] P. Mulders, Phys. Rept. 185, 83 (1990).

[40] L.L. Frankfurt and M.I. Strikman, Phys. Rept. 160, 235 (1988).

[41] M. Ropele, M. Traini and V. Vento, Nucl. Phys. A 584, 634 (1995).

[42] L. Mankiewicz, G. Piller, and T. Weigl, Eur. Phys. J. C 5, 119 (1988).

[43] S. Scopetta, Phys. Rev. C 70, 015205 (2004).

[44] C. Ciofi degli Atti, S. Scopetta, E. Pace, and G. Salmè, Phys. Rev. C48, R968 (1993).

[45] C. Ciofi degli Atti, E. Pace, and G. Salmè, Phys. Rev. C46, R1591 (1992). 
[46] F. Cano and B. Pire, Nucl. Phys. A 711, 133c (2002); Eur. Phys. J. A 19, 423 (2004).

[47] V. Guzey and M.I. Strikman, Phys. Rev. C 68, 015204 (2003).

[48] R. L. Heimann, Nucl. Phys. B64 (1973) 429; J. Ellis and M. Karliner, Phys. Lett. B213 (1988) 73 .

[49] R. Carlitz and J. Kaur, Phys. Rev. Lett. 38, 673 (1977); J. Kaur, Nucl. Phys. B 128, 219 (1977); A. Schaefer, Phys. Lett. B 208, 175 (1988).

[50] G. Altarelli, R.D. Ball, S. Forte and G. Ridolfi, Nucl. Phys. B 496, 337 (1997).

[51] M.M. Giannini, Rep. Prog. Phys. 54, 453 (1991).

[52] L.A. Ahrens et al., Phys. Rev. D 35, 785 (1987).

[53] R. Bijker, F. Iachello and A. Leviatan, Ann. Phys. 236, 69 (1994); Phys Rev. C 54, 1935 (1996); Phys. Rev D 55, 2862 (1997).

[54] W. Furmansky and R. Petronzio, Z. Phys. C 11, 293 (1982).

[55] R.D. Ball, S. Forte and G. Ridolfi, Phys. Lett. B 378, 255 (1996).

[56] R. Mertig, W. L. van Neerven, Z.Phys. C70, 637 (1996); W. Vogelsang, Phys. Rev D54, $2023(1996)$.

[57] G. Altarelli and G. G. Ross, Phys. Lett. B 212, 391 (1988).

[58] F.E. Close and R.G. Roberts, Phys. Lett. B 316, 165 (1993).

[59] Particle Data Group, J. Ashman et al., Phys. Rev. D 54, 1 (1996).

[60] EMC Collaboration, J. Ashman et al., Nucl. Phys. B 328, 1 (1989) .

[61] SMC Collaboration, D. Adams et al., Phys. Rev. D 56, 5330, (1997).

[62] E154 Collaboration, K. Abe et al., Phys. Rev. Lett. 79, 26 (1997).

[63] S. Noguera, S. Scopetta and V. Vento, Phys. Rev. D70, 094018 (2004). 
[64] M. Diehl, Th. Feldmann, R. Jakob, and P. Kroll, hep-ph/0408173.

[65] M. Guidal, M.V. Polyakov, A.V. Radyushkin, and M. Vanderhaeghen, hep-ph/0410251.

[66] J. Blümlein and M. Böttcher, Nucl. Phys. B 636, 225 (2002). 


\section{FIGURE CAPTIONS}

Fig. 1: The handbag contribution to the DVCS process in the present approach.

Fig. 2: The GPD $\tilde{H}$ for the flavor $u$, for $\Delta^{2}=-0.1 \mathrm{GeV}^{2}$ and $\xi=0.1$, at the momentum scale of the model. Dashed curve: result in the pure Isgur and Karl model, Eq. (85). The small tails which are found in the forbidden regions, $x \leq \xi$ and $x \geq 1$, represent the amount of support violation of the approach. Full curve: the complete result of the present approach, Eq. (38). The two curves are obtained in correspondence of the two extreme values of the Regge intercept [48], $a_{f}=0$ (upper curve at low $x$ ) and $a_{f}=0.5$.

Fig. 3: For the $\xi$ values which are allowed at $\Delta^{2}=-0.1 \mathrm{GeV}^{2}, \tilde{H}_{u}\left(x, \xi, \Delta^{2}\right)$, evaluated using our main equation, Eq. (38), is shown for four different values of $x$, at the momentum scale of the model. From top to bottom, the dash-dotted line represents the GPD at $x=0.05$, the full line at $x=0.1$, the dashed line at $x=0.2$, and the long-dashed line at $x=0.4$. The curves are obtained in correspondence of the value of the Regge intercept $a_{f}=0.5$ (see text).

Fig. 4: The $x$ and $\xi$ dependences of $\tilde{H}_{u}\left(x, \xi, \Delta^{2}\right)$, for $\Delta^{2}=-0.1 \mathrm{GeV}^{2}$, at the momentum scale of the model. The value of the Regge intercept has been taken to be $a_{f}=0.5$ (see text).

Fig. 5: We show the structure function $x g_{1}^{p}\left(x, Q^{2}\right)$ obtained at $Q^{2}=10 \mathrm{GeV}^{2}$ by evolving at NLO the model calculation [24] considering the structure of the constituents, for the two extreme Regge behaviors mentioned in the text $\left(a_{f}=0\right.$ is the upper curve, here and also in the following figure). The data from refs. [60] (full dots) and [61] at $Q^{2} \approx 10 \mathrm{GeV}^{2}$ are also shown.

Fig. 6: The structure function $x g_{1}^{n}\left(x, Q^{2}\right)$ for the neutron evolved at NLO to $Q^{2}=5 \mathrm{GeV}^{2}$, for the two extreme Regge behaviors mentioned in the text, is shown by the two full curves. 
The data from refs. [62] at $Q^{2}=5 \mathrm{GeV}^{2}$ are also shown.

Fig. 7: Comparison of the results of the present approach for the valence distribution $\tilde{H}_{u}^{v}\left(x, 0, \Delta^{2}\right)$ (full curves), evaluated at $\Delta^{2}=-0.1 \mathrm{GeV}^{2}$, evolved to NLO from the hadronic scale up to $Q^{2}=4 \mathrm{GeV}^{2}$, with the ansatz proposed in [64] (dotted curve), evaluated in the same kinematical conditions. The two full curves are obtained in correspondence of the two extreme values of the Regge intercept [48], $a_{f}=0$ (upper curve at low $x$ ) and $a_{f}=0.5$. 


\section{FIGURES}

FIG. 1.

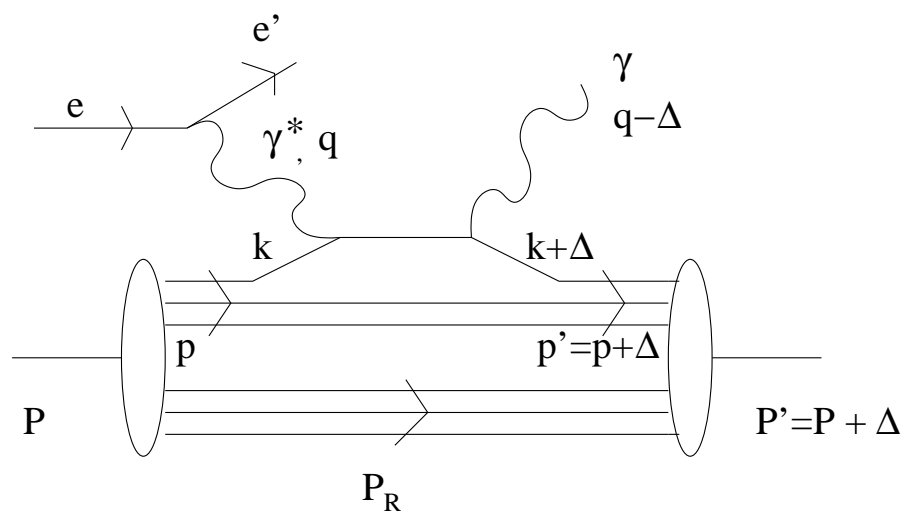


FIG. 2.

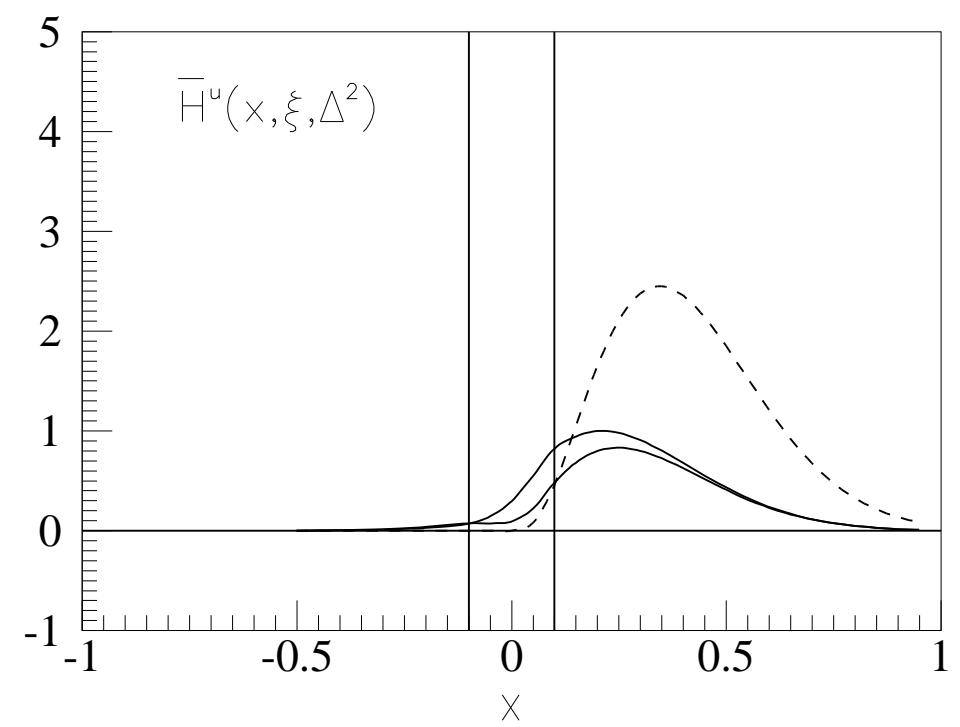


FIG. 3 .

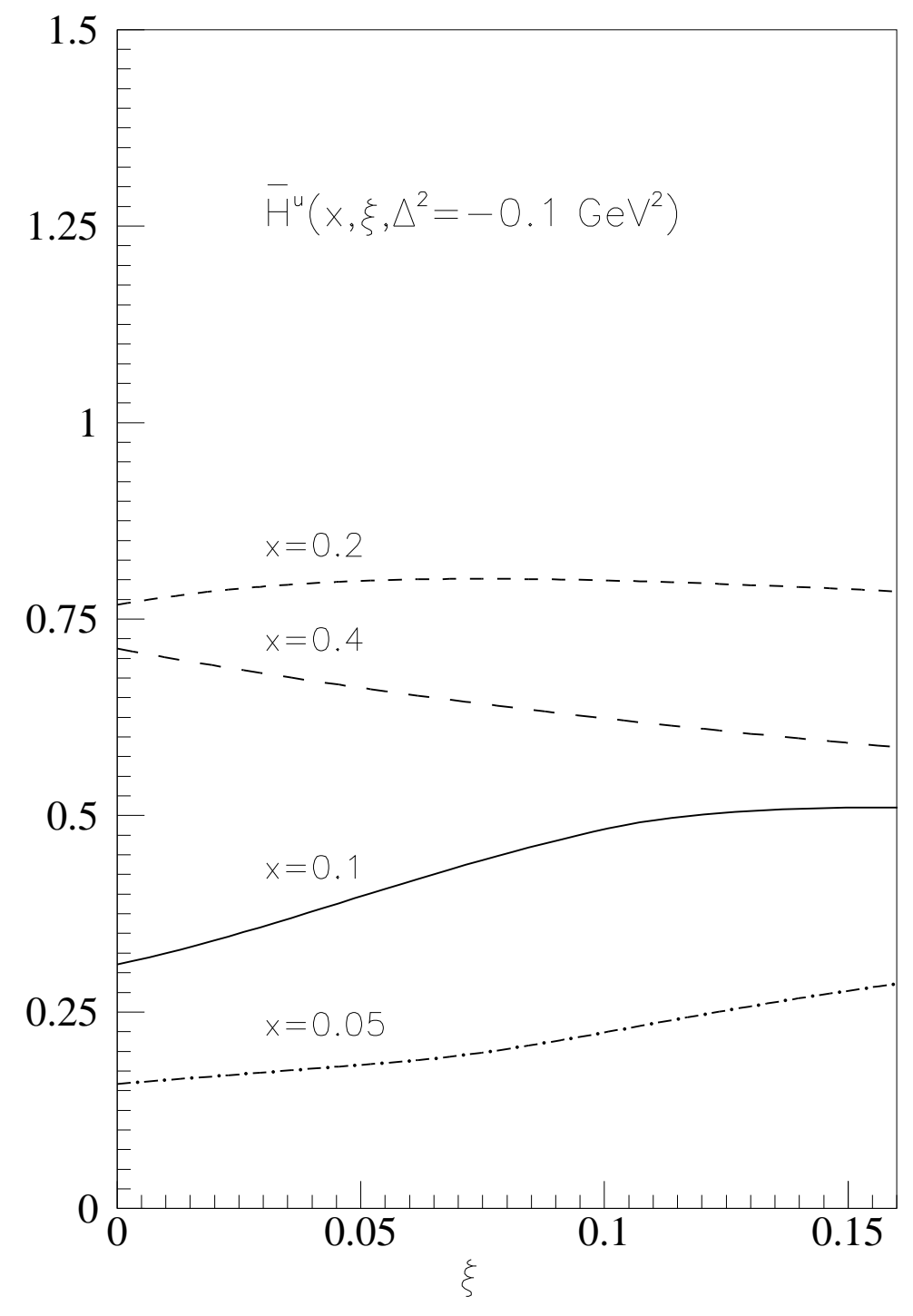


FIG. 4.

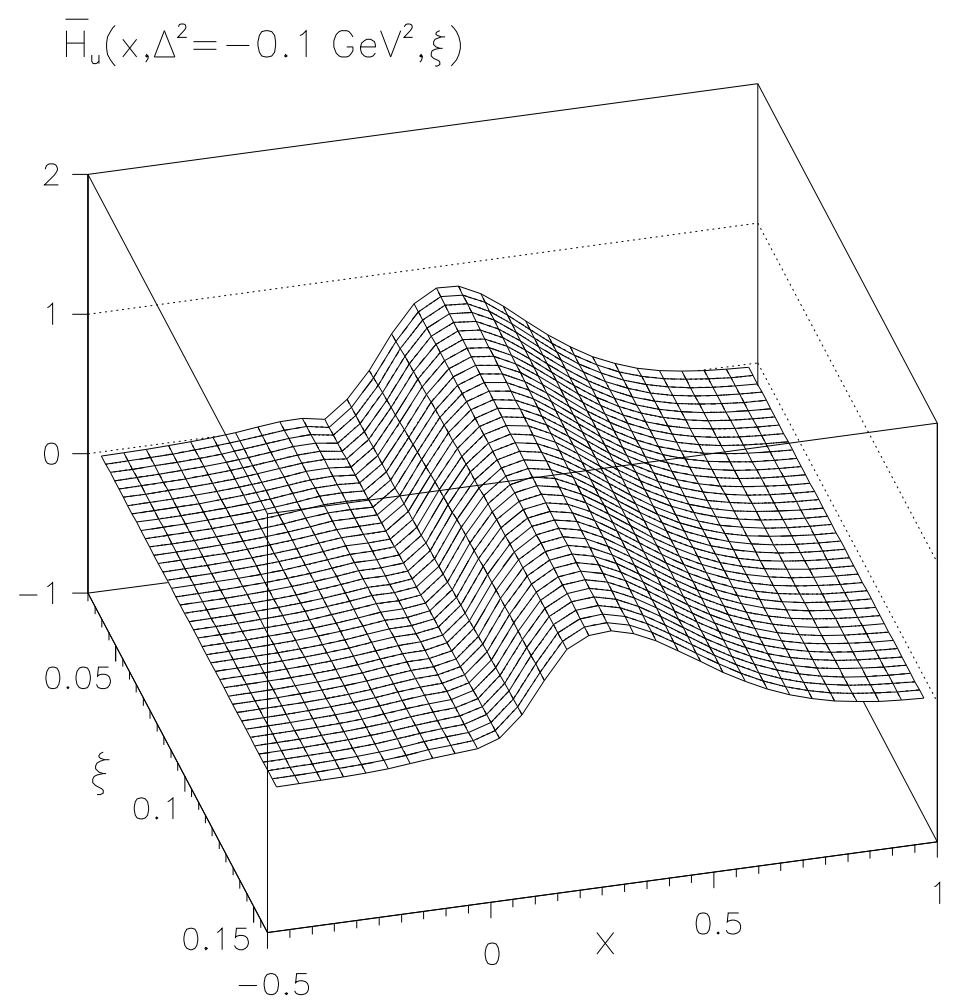


FIG. 5.

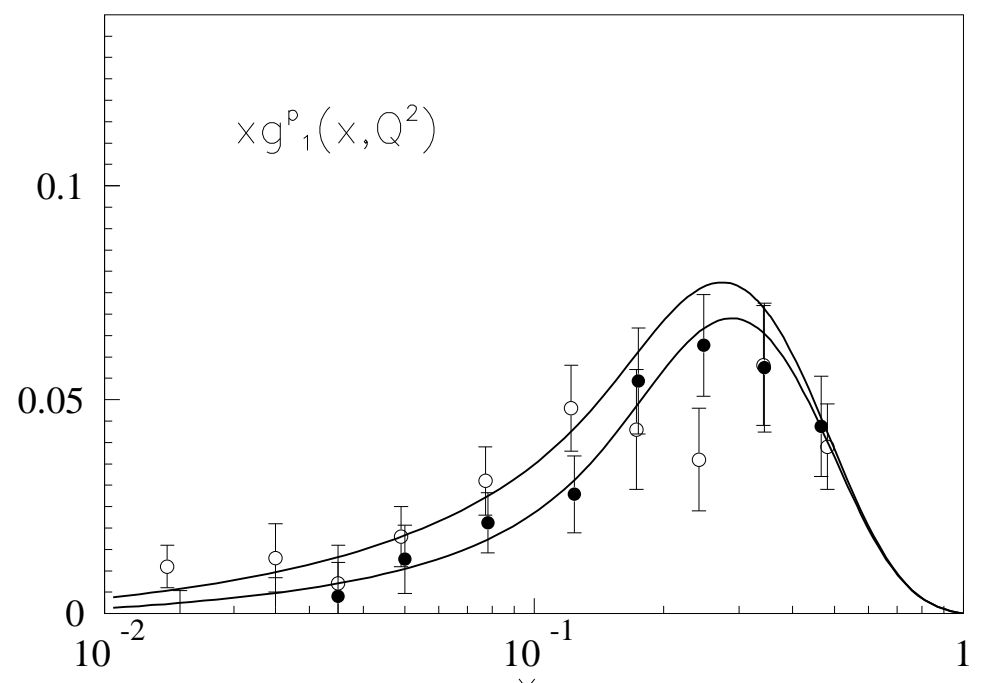


FIG. 6.

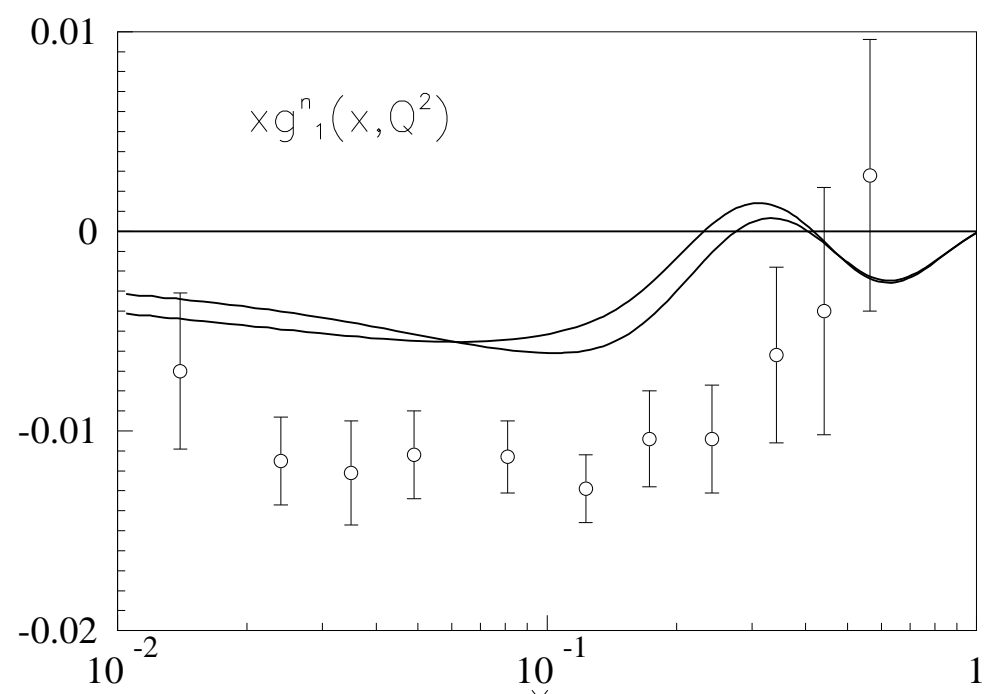


FIG. 7.

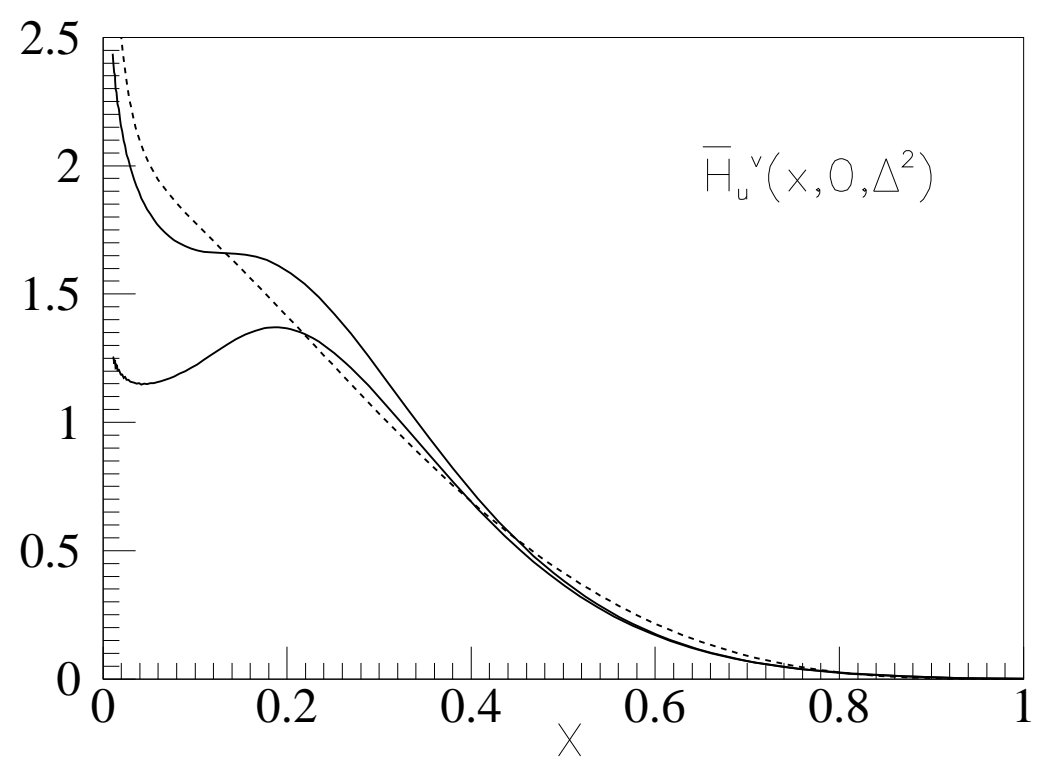

